\title{
ON THE TWODIMENSIONAL STEADY-STATE PROBLEM OF A VISCOUS GAS IN AN EXTERIOR DOMAIN
}

\author{
G.P. Galdi, A. Novotný And M. Padula
}

\begin{abstract}
We investigate plane steady flows of a viscous, isothermal, compressible fluid past an obstacle, with nonzero velocity at infinity. Using the decomposition of the velocity field onto its compressible and incompressible parts, we prove an existence and uniqueness theorem, under the assumption that the external data are "sufficiently small".
\end{abstract}

\section{Introduction.}

Over the last ten years, a great interest has been devoted to the study of the Navier-Stokes equations in exterior domains for the incompressible case (see e.g. [3], [4], [8], [9], [10], [14], [15], [21], [24] (Vol. I, Chapters 5, 7, Vol. II, Chapters. 9, 10), [27], [31], [32], [35], [40], [41], [47], [56]) and, more recently, also for the compressible case (see [37], [38], [39], [36], [44], [45], [48]). In general, exterior fluid dynamical problems present further essential difficulties compared to the interior ones (bounded domains). In the incompressible case, this was first pointed out by J. Leray in his celebrated paper of 1934, cf. [34]. Actually, for flow past an obstacle, in spite of great effort of prominent mathematicians, there are several basic questions which are yet to be answered. In particular, existence and uniqueness of plane flows offers several unsolved and challenging features.

The present paper is devoted to the investigation of the steady compressible isothermal flows (governed by Poisson-Stokes equations) in twodimensional exterior domains.

In order to describe the difficulty of the problem, we wish to briefly recall the main results which are known for incompressible fluids. In this case, we have the following Navier-Stokes equations

$$
\begin{gathered}
-\mu \Delta \mathbf{v}+\nabla p=f-\operatorname{div}(\mathbf{v} \otimes \mathbf{v}), \quad \operatorname{div} \mathbf{v}=0 \\
\left.\mathbf{v}\right|_{\partial \Omega}=0, \quad \mathbf{v}(x) \rightarrow \mathbf{v}_{\infty} .
\end{gathered}
$$

Here $\mathbf{v}, p$ are unknown velocity and pressure, respectively, $f$ is a (given) external force, $\mu>0$ is the (constant) viscosity of the fluid and $\mathbf{v}_{\infty}$ is a 
given constant velocity at infinity. Moreover, $\Omega$ is a domain exterior to a compact region (with sufficiently smooth boundary).

The steady flows in threedimensional and twodimensional exterior domains are qualitatively very different as well as different are the contributions furnished towards their mathematical resolution. Actually, in three dimensions, there is an exhausting mathematical theory including a very detailed description of the asymptotic properties of solutions. This investigation starts with the fundamental work of Leray [34], where he shows the solvability of (1.1) for data of arbitrary size in the class of solutions having the finite Dirichlet integral:

$$
\int_{\Omega}|\nabla \mathbf{v}|^{2} d x<+\infty
$$

The study of Leray's solutions and of their asymptotic structure has been successively continued and completed by the works of Fujita [21], Finn [17], [18], [19], Clark [13], Babenko [5], Galdi [23], [24], [25], Farwig [16], Heywood [30], Novotny and Padula [47] and others. However, in the twodimensional case, the literature furnishes much less complete results. Specifically, as in the threedimensional case, Leray proved the existence of a solution to $(1.1)_{1-3}$, for large data, having the finite Dirichlet integral (1.2), but he did not succeed in showing that such a solution tends to the prescribed velocity at infinity. This is due to the fact that the condition (1.2) is not sufficient (unlike threedimensional case) to control, even in a very weak sense, the behaviour at infinity of the function $\mathbf{v}$ itself ${ }^{1}$. This problem remains still open, only partial answers having been given. Precisely, Gilbarg and Weinberger [28], [29] and Amick [3] proved that, if $f=0$, for any Leray's solution there exists a constant vector $\mathbf{v}_{0}$ such that $\mathbf{v} \rightarrow \mathbf{v}_{0}$ at infinity; if $\mathbf{v}_{0} \not \equiv 0$ then the convergence is in the mean, otherwise being pointwise and uniform. However, they do not prove whether $\mathbf{v}_{0}=\mathbf{v}_{\infty}$. Furthermore, Amick [4], using an auxiliary result of Smith [55], shows that if $\mathbf{v}_{0} \not \equiv 0$ and $\mathbf{v} \rightarrow \mathbf{v}_{0}$ uniformly pointwise, then $\mathbf{v}(x)=\mathbf{v}_{0}+O\left(|x|^{-1 / 2}\right)$. By using a different method, Finn and Smith [20] proved for $\mathbf{v}_{\infty} \not \equiv 0$ existence and uniqueness of solutions for $f \equiv 0$ and small $\mathbf{v}_{\infty}$ such that $\mathbf{v}(x)=\mathbf{v}_{\infty}+O\left(|x|^{-1 / 2}\right)$. Several attempts have also been made in order to apply fixed point theory in suitable $L^{q}$ spaces. In this respect, there has been an intensive study for corresponding linearized systems (that is Stokes-like, if $\mathbf{v}_{\infty}=0$, or Oseen-like, if $\mathbf{v}_{\infty} \not \equiv 0$ ), cf. Galdi and Simader [27], Galdi [23], [24], Kozono and Sohr [32], Borchers, Miyakawa [8]. However, these studies have revealed, so far, insufficient to produce results for the nonlinear theory, at least when $\mathbf{v}_{\infty}=0$, because of

\footnotetext{
${ }^{1}$ It is simple to give examples of functions with finite Dirichlet integral which diverge at infinity, see e.g. Galdi's book [24].
} 
the difficulty of estimating the nonlinear term. Very recently, Galdi [24], [26] has performed a detailed study of existence, uniqueness and corresponding estimates of solutions to the linearized Oseen problem in suitable homogeneous Sobolev spaces. This study allows him to dominate the nonlinear terms and to obtain, for $\mathbf{v}_{\infty} \not \equiv 0$ and small external data $\left(f, \mathbf{v}_{\infty}\right)$, existence and uniqueness to the full nonlinear problem (1.1).

Up to now, no attempt to investigate the plane steady flow of a viscous compressible fluid past a body is known in the literature. Even in the simpler threedimensional case, the problem received a satisfactory answer only recently through the work of Novotný and Padula [45]. In that paper, the authors propose the following method of decomposition. The linearized compressible system (see Section 2) is split into three auxiliary problems: i) a Neumann problem for the Laplacian, governing essentially the compressible part of the velocity field; ii) an Oseen system, governing its incompressible part and iii) a transport equation governing the density.

This method (see Section 3) along with the recent contribution of Galdi mentioned above [24] (see Section 6) makes it possible to prove existence and uniqueness also for the twodimensional exterior flows of a compressible fluid $^{2}$, at least for small external force and small but nonzero velocity at infinity (see Theorems 5.1 and 5.2). This is the main achievement of the present work.

The paper is organized as follows:

1. Introduction

2. Equations

3. The Decomposition Method

4. Functional Spaces

5. Main Theorems (Theorem 5.1, Theorem 5.2)

6. Preliminary Results

7. Proof of Theorem 5.2

8. $\quad$ Proof of Theorem 5.1

Appendix

References

Acknowledgement. G. P. Galdi and M. Padula thank the G.N.F.M. of the

\footnotetext{
${ }^{2}$ In fact, we consider only the isothermal case. Clearly, the similar results, with evident modifications, hold for the barotropic flows, provided the constitutive function for the pressure is "sufficiently" regular and with positive derivative, cf. e.g. [48]. It is likely that the same result is valid also for the heat conductive case. But, unlike bounded domains, this generalization to the exterior domains is by no means obvious, cf. Novotný and Penel [48] (for the threedimensional case).
} 
Italian C.N.R. and $40 \%$ and $60 \%$ contracts with MURST. The work of A. Novotný was partially supported by G.N.F.M. of the Italian C.N.R., during his stay in Ferrara. The authors thank the referee for helpful suggestions.

\section{Equations.}

We consider a viscous steady isothermal flow of a perfect gas in a twodimensional exterior domain $\Omega \subset \mathbb{R}^{2}$; it is governed by the following system of equations for unknown functions $\mathbf{v}=\left(\mathbf{v}_{1}, \mathbf{v}_{2}\right)$ (the velocity) and $\rho$ (the density):

$$
\begin{gathered}
-\mu_{1} \Delta \mathbf{v}-\left(\mu_{1}+\mu_{2}\right) \nabla \operatorname{div} \mathbf{v}+\nabla \rho=\rho f-\operatorname{div}(\rho \mathbf{v} \otimes \mathbf{v}) \\
\operatorname{div}(\rho \mathbf{v})=0
\end{gathered}
$$

Here $\mu_{1}, \mu_{2}$ are shear and bulk viscosities which are supposed to be constants satisfying the relations

$$
\mu_{1}>0, \mu_{1}+\mu_{2} \geq 0
$$

and $f$ is the density of the external body forces. The boundary conditions and conditions at infinity are

$$
\begin{aligned}
\left.\mathbf{v}\right|_{\partial \Omega} & =0 \\
\mathbf{v}(x) \rightarrow \mathbf{v}_{\infty}, \quad \rho(x) & \rightarrow 1 \quad \text { as }|x| \rightarrow \infty
\end{aligned}
$$

Here $\mathbf{v}_{\infty} \neq 0$ is a nonzero constant vector which can be taken (without loss of generality) in the direction of $x_{1}$-axis, i.e. $\mathbf{v}_{\infty}=\left(v_{\infty}, 0\right), v_{\infty} \in \mathbb{R}^{1}$. The equations for the perturbations

$$
v=\mathbf{v}-\mathbf{v}_{\infty}, \quad \sigma=\rho-1
$$

read

$$
\begin{gathered}
-\mu_{1} \Delta v-\left(\mu_{1}+\mu_{2}\right) \nabla \operatorname{div} v+\nabla \sigma+v_{\infty} \frac{\partial v}{\partial x_{1}}=F(\sigma, v), \\
\operatorname{div} v+\operatorname{div}\left(\sigma\left(v+\mathbf{v}_{\infty}\right)\right)=0 \\
\left.v\right|_{\partial \Omega}=-\mathbf{v}_{\infty} \\
v(x) \rightarrow 0, \sigma(x) \rightarrow 0 \text { as }|x| \rightarrow \infty
\end{gathered}
$$

where

$$
F(\sigma, v)=(1+\sigma) f-\operatorname{div}((1+\sigma) v \otimes v)-v_{\infty} \frac{\partial(\sigma v)}{\partial x_{1}}
$$


Therefore, it is useful to investigate first the linearized system

$$
\begin{gathered}
-\mu_{1} \Delta v-\left(\mu_{1}+\mu_{2}\right) \nabla \operatorname{div} v+\nabla \sigma+v_{\infty} \frac{\partial v}{\partial x_{1}}=\mathcal{F}, \\
\operatorname{div} v+\operatorname{div}\left(\sigma\left(w+\mathbf{v}_{\infty}\right)\right)=0 \\
\left.v\right|_{\partial \Omega}=-\mathbf{v}_{\infty}, \\
v(x) \rightarrow 0, \sigma(x) \rightarrow 0 \text { as }|x| \rightarrow \infty
\end{gathered}
$$

where the unknown functions are $\sigma, v$ while $w, \mathcal{F}$ are given quantities. Problem $\left(2.4^{\prime}\right)$ with $\mathbf{v}_{\infty} \neq 0$ in exterior threedimensional domains was investigated by Novotný and Padula [45] (see also Novotný [43], [44], Padula [49], [50], Novotný and Penel [48]). ${ }^{3}$ However, to our knowledge, no results are available in twodimensional case.

It is useful, for our purposes, to consider the following system which is slightly more general than $\left(2.4^{\prime}\right)$

$$
\begin{gathered}
-\mu_{1} \Delta v-\left(\mu_{1}+\mu_{2}\right) \nabla \operatorname{div} v+\nabla \sigma+v_{\infty} \frac{\partial v}{\partial x_{1}}=\mathcal{F}, \\
\operatorname{div} v+\operatorname{div}\left(\sigma\left(w+\mathbf{v}_{\infty}\right)\right)=g, \\
\left.v\right|_{\partial \Omega}=-\Psi \\
v(x) \rightarrow 0, \sigma(x) \rightarrow 0 \text { as }|x| \rightarrow \infty
\end{gathered}
$$

This system presents two major difficulties: 1) the well known difficulties of 2-D elliptic exterior problems (similar to those met in all linearizations of the incompressible Navier-Stokes equations); 2) the difficulties related to its mixed elliptic-hyperbolic character (which is characteristic for all linearizations of compressible Navier-Stokes equations). The analysis of (2.4) in two dimensions differs essentially from that one in the 3-D case. Unlike three dimensions, we need very sharp estimates "anisotropic in space" and "nonhomogenous" in $\mathbf{v}_{\infty}$. The form of these estimates is conjectured by the well known structure of 2-D Oseen tensor (especially by its summability and rescaling properties), and from what is known for 2-D exterior Oseen problem. In this respect, the splitting inspired by [45] (called the decomposition method), plays an essential role.

The investigation of the fully nonlinear system (2.3) for small data is carried out by a perturbation method. Unlike 3-D case, the "anisotropic"

\footnotetext{
3 For the sake of completness, we recall that the literature for two- and threedimensional bounded domains is more extended, cf. Padula [51], Valli [57], Beirão da Veiga [6], Farwig [16], Novotný and Padula [46] and the references quoted there.
} 
estimates with the precise dependence on $\mathbf{v}_{\infty}$ for the system $\left(2.4^{\prime}\right)$ along with the correct understanding of the internal structure of the nonlinearity $(2.3)_{2}$, are inevitable for performing the fixed point argument.

\section{The Decomposition Method.}

To solve the nonlinear problem, we apply a Banach fixed point theorem (see e.g., Zeidler [58]) to the nonlinear operator

$$
\mathcal{T}:(\tau, w) \rightarrow F(\tau, w) \rightarrow(\sigma, v)
$$

where $(\sigma, v)$ is a solution of the linear system (2.4) corresponding to $w$, and $\mathcal{F}=F(\tau, w), \Psi=\mathbf{v}_{\infty}, g=0$.

To show existence of solutions for the linear system (2.4), we use the following decomposition method introduced by Novotný and Padula [45]:

We look for $v$ in the form

$$
\begin{gathered}
v=u+\nabla \varphi, \\
\operatorname{div} u=0,\left.\quad u \cdot \nu\right|_{\partial \Omega}=0, \\
\left.\frac{\partial \varphi}{\partial \nu}\right|_{\partial \Omega}=-\Psi \cdot \nu,
\end{gathered}
$$

with $\nu$ the outer normal to $\partial \Omega$.

For a fixed $w, \Psi$, and $g$, we define a formal linear map

$$
\mathcal{L}: \tau \rightarrow \sigma
$$

as follows:

(i) For a given $\tau$ we solve a Neumann problem

$$
\begin{gathered}
\Delta \varphi=g-\operatorname{div}\left(\tau\left(w+\mathbf{v}_{\infty}\right)\right), \\
\left.\frac{\partial \varphi}{\partial \nu}\right|_{\partial \Omega}=-\Psi \cdot \nu, \quad \nabla \varphi(x) \rightarrow 0 \text { as }|x| \rightarrow \infty,
\end{gathered}
$$

for the unknown function $\varphi$.

(ii) Once $\varphi$ is known, we solve for a given $\mathcal{F}$, the nonhomogenous Oseen problem for the unknown functions $(\Pi, u)$

$$
\begin{gathered}
-\mu_{1} \Delta u+\nabla \Pi+v_{\infty} \frac{\partial u}{\partial x_{1}}=\mathcal{F}, \\
\operatorname{div} u=0, \\
\left.u\right|_{\partial \Omega}=-\left.(\Psi+\nabla \varphi)\right|_{\partial \Omega}, \\
u(x) \rightarrow 0, \Pi(x) \rightarrow 0 \text { as }|x| \rightarrow \infty .
\end{gathered}
$$


(iii) Then, given $\Pi$ and $\varphi$ and $w, \sigma$ is found as a solution of the transport equation:

$$
\begin{gathered}
\sigma+\left(2 \mu_{1}+\mu_{2}\right) \operatorname{div}\left(\sigma\left(w+\mathbf{v}_{\infty}\right)\right)=\left(2 \mu_{1}+\mu_{2}\right) g+\Pi-v_{\infty} \frac{\partial \varphi}{\partial x_{1}} \\
\sigma(x) \rightarrow 0 \text { as }|x| \rightarrow \infty
\end{gathered}
$$

If a "sufficiently smooth" fixed point $\sigma$ of $\mathcal{L}$ exists, then $\sigma$ and the corresponding field $v=u+\nabla \varphi$ satisfy the original system (2.4), as the reader can easily verify. Once the estimates for the linear auxiliary problems (3.4), (3.5), and (3.6) are obtained, the proof of the existence of a fixed point relies on the following fundamental considerations:

(i) The term $\operatorname{div}\left(\sigma\left(w+\mathbf{v}_{\infty}\right)\right)$ in (3.6) contains the same order of differentiation for $\sigma$ as the leading term in the equations; therefore, it cannot be considered as a perturbation; rather, it has to be treated as a linear term.

(ii) In the transport equation (3.6), the quantities $\sigma$ and $\operatorname{div}\left(\sigma\left(w+\mathbf{v}_{\infty}\right)\right)$ have the same summability and regularity; therefore, for a given $w$, the field $\tau$ should be chosen in such a way that its regularity coincides with that of $\operatorname{div}\left(\tau\left(w+\mathbf{v}_{\infty}\right)\right)$.

(iii) If

$$
\left.\left(w+\mathbf{v}_{\infty}\right)\right|_{\partial \Omega}=0,\left.\quad(\operatorname{div} w)\right|_{\partial \Omega}=0,\left.\quad g\right|_{\partial \Omega}=0
$$

then (by $(2.4)_{2}$ ) we have also

$$
\left.\left(\operatorname{div}\left(\sigma\left(w+\mathbf{v}_{\infty}\right)\right)\right)\right|_{\partial \Omega}=0,\left.\quad(\operatorname{div} v)\right|_{\partial \Omega}=0 .
$$

For this choice of $w$, the second derivatives of $\operatorname{div}\left(\sigma\left(w+\mathbf{v}_{\infty}\right)\right)$ can be estimated only by the Laplacian of $\operatorname{div}\left(\sigma\left(w+\mathbf{v}_{\infty}\right)\right)$.

(iv) Equation (3.5) yields $\Delta \Pi=\operatorname{div} \mathcal{F}$; hence, $\Delta \Pi$ can be estimated only in terms of the right hand side.

Remark 3.1. Similarly as in the case of flow in bounded domains and in the analogy with the incompressible case (Finn [17]), the results we shall find here can be easily generalized to the nonhomogenous boundary condition for the velocity:

$$
\left.\mathbf{v}\right|_{\partial \Omega}=\mathbf{v}^{*},
$$

provided $\mathbf{v}^{*}$ is sufficiently small in an appropriate norm and $\left.\mathbf{v}^{*} \cdot \nu\right|_{\partial \Omega}=0 .{ }^{4}$

\footnotetext{
${ }^{4}$ Details can be found in the paper of Novotný and Penel [48], who investigate the same situation for heat conducting fluids in threedimensional exterior domains.
} 


\section{Functional Spaces.}

Let $\Omega \subset \mathbb{R}^{2}$ be an exterior domain such that $\bar{\Omega}_{c}=\mathbb{R}^{2} \backslash \Omega$ is a compact set (the superposed bar denotes the closure). Without loss of generality, we assume $0 \in \Omega_{c}$, interior of $\bar{\Omega}_{c}$. The boundary $\partial \Omega$ of $\Omega$ is of class $\mathcal{C}^{k+3}, k=0,1, \ldots$ and its outer unit normal is denoted by $\nu$. By $B_{R}$, we denote a ball in $\mathbb{R}^{2}$ with radius $R$ and center at 0 and set $\Omega_{R}=B_{R}-\bar{\Omega}_{c}$ (provided $\Omega_{c} \subset B_{R}$ ), $\Omega^{R}=B^{R}=\mathbb{R}^{2}-B_{R}$.

We use the following functional spaces:

$\mathcal{C}_{0}^{\infty}(\Omega)$ is the space of smooth functions with compact support in $\Omega$ and $\mathcal{C}_{0}^{\infty}(\bar{\Omega})$ is the class of restrictions to $\Omega$ of functions from $\mathcal{C}_{0}^{\infty}\left(\mathbb{R}^{2}\right)$. $\mathcal{C}^{k}(\bar{\Omega})(k=0,1, \ldots)$ is the Banach space of continuous functions with continuous derivatives in $\bar{\Omega}$ up to the order $k$; it is equipped with the usual norm $|u|_{\mathcal{C}^{k}}=\sup _{x \in \Omega, \alpha=1, \ldots, k}\left|\nabla^{\alpha} u(x)\right|$.

$L^{t}(\Omega)=W^{0, t}(\Omega), 1 \leq t \leq \infty$, is the usual Lebesgue space with the norm $\|\cdot\|_{0, t}$, and $W^{k, t}(\Omega), 1<t<\infty, k=1,2, \ldots$ is the Sobolev space with the norm $\|\cdot\|_{k, t}=\sum_{m=0}^{k}\left\|\nabla^{m} \cdot\right\|_{0, t}$. Moreover, $W_{0}^{k, t}(\Omega)$ is the subspace of $W^{k, t}(\Omega)$ constituted by functions having zero traces at the boundary $\partial \Omega$.

$\hat{H}_{0}^{1, t}(\Omega)={\overline{\mathcal{C}_{0}^{\infty}}(\Omega)}^{|\cdot|_{1, t}}$ is the Banach space with norm $|\cdot|_{1, t}=\|\nabla \cdot\|_{0, t}$, $1<t<+\infty$; here and in the sequel, the supperposed bar with a norm denotes the completion in that norm.

$\mathcal{H}_{0}^{k, t}(\Omega)=\overline{\mathcal{C}}_{0}^{\infty}(\bar{\Omega}){ }^{\|\nabla \cdot\|_{k-1, t}} \cap \hat{H}_{0}^{1, t}(\Omega)$ is the Banach space with the norm $\|\nabla \cdot\|_{k-1, t}, 1<t<+\infty$.

$\hat{H}_{\infty}^{1, t}(\Omega)={\overline{\mathcal{C}_{0}^{\infty}(\bar{\Omega})}}^{|\cdot|_{1, t}}$ is the Banach space with the norm $|\cdot|_{1, t}, 1<t<$ $\infty$. If $2 \leq t<+\infty$ then the elements of $\hat{H}_{\infty}^{1, t}(\Omega)$ are equivalence classes $\{u+c\}_{c \in \mathbb{R}^{1}}, u \in L^{t}{ }_{\text {loc }}(\Omega), \nabla u \in L^{t}(\Omega)$. If $1<t<2$ then the elements of $\hat{H}_{\infty}^{1, t}(\Omega)$ are such that $u \in L^{2 t /(2-t)}(\Omega), \nabla u \in L^{t}(\Omega)$, cf. Lemma 4.1.

Similarly, $\mathcal{H}_{\infty}^{k, t}(\Omega)={\overline{\mathcal{C}_{0}^{\infty}(\bar{\Omega})}}^{\|\nabla \cdot\|_{k-1, t}}, 1<t<\infty$ is the Banach space with the norm $\|\nabla \cdot\|_{k-1, t}$.

The dual space to $\hat{H}_{0}^{1, t^{\prime}}(\Omega), t^{-1}+t^{-1}=1$ is denoted by $\hat{H}^{-1, t}(\Omega)$ and its norm is given by $|\cdot|_{-1, t}$, the dual space to $\hat{H}_{\infty}^{1, t^{\prime}}(\Omega)$ is denoted by $\left(\hat{H}_{\infty}^{1, t^{\prime}}(\Omega)\right)^{*}$ and the corresponding norm is given by $|\cdot|_{*, t}$, the dual space to $W_{0}^{1, t^{\prime}}(\Omega)$ is denoted by $W^{-1, t}(\Omega)$ and its norm is expressed by $\|\cdot\|_{-1, t}$.

For a detailed description of these spaces and their properties, see Simader and Sohr [53], Simader [52], Galdi [24].

If not stated explicitly, all norms we use in the paper refer to $\Omega$, e.g., $\|\cdot\|_{0, t}$ is a norm in $L^{t}(\Omega)$. If a norm refers to another domain, say $G$, then 
we denote it explicitly by using an additional index in the symbol of that norm, e.g., $\|\cdot\|_{0, t, G}$ is the norm in $L^{t}(G)$ or $|\cdot|_{\mathcal{C}^{k}, G}$ denotes the norm in $\mathcal{C}^{k}(\bar{G})$. We do not distinguish in the notation between the spaces of scalar and vector valued functions, e.g. $W^{k, t}(\Omega)$ means either $W^{k, t}\left(\Omega ; \mathbb{R}^{1}\right)$ or $W^{k, t}\left(\Omega ; \mathbb{R}^{2}\right)$. The difference is always clear from the context.

If not stated explicitely, by $c, c^{\prime}, c_{i}, c_{i}^{\prime}, i \geq 0$, we denote positive constants which depend at most of $k, q, p$ (eventually $t$ ), of $\partial \Omega$ and of $\lambda_{0}$ (where $\lambda_{0}$ is the upper bound for the prescribed velocity at infinity $\mathbf{v}_{\infty}: 0<\left|\mathbf{v}_{\infty}\right|<\lambda_{0}$ ). In particular, they are independent of $\mathbf{v}_{\infty}$. The symbols $c, c^{\prime}$ may be used, even in the same estimate, to indicate distinct constants.

In the sequel, it will be of some convenience, to introduce the following Banach spaces:

$$
\begin{aligned}
\mathbf{Z}= & \left\{(\sigma, u): u \in \mathcal{H}_{\infty}^{k+2, p}(\Omega) \cap L^{3 q /(3-2 q)}(\Omega), \nabla u \in L^{3 q /(3-q)}(\Omega),\right. \\
& \nabla^{2} u \in W^{1, q}(\Omega), \quad \frac{\partial u}{\partial x_{1}} \in L^{q}(\Omega), \quad u_{2} \in L^{2 q /(2-q)}(\Omega), \\
& \left.\nabla u_{2} \in L^{q}(\Omega), \sigma \in L^{2 q /(2-q)}(\Omega) \cap W^{k+1, p}(\Omega), \nabla \sigma \in W^{1, q}(\Omega)\right\}
\end{aligned}
$$

with $1<q<3 / 2, p>2$ and $k=1,2, \ldots$; it is equipped with the following norm

$$
\begin{aligned}
\|(\sigma, u)\|_{\mathbf{z}, \lambda}= & \lambda^{2 / 3}\|u\|_{0,3 q /(3-2 q)}+\lambda^{1 / 3}\left(\|\nabla u\|_{0,3 q /(3-q)}+\left\|\nabla^{2} u\right\|_{1, q}\right. \\
& \left.+\|\sigma\|_{0,2 q /(2-q)}+\|\nabla \sigma\|_{1, q}+\|\nabla u\|_{k+1, p}+\|\sigma\|_{k+1, p}\right) \\
& +\lambda\left(\left\|\frac{\partial u}{\partial x_{1}}\right\|_{0, q}+\left\|\nabla u_{2}\right\|_{0, q}+\left\|u_{2}\right\|_{0,2 q /(2-q)}\right)
\end{aligned}
$$

with $\lambda$ a positive parameter. The set

$$
Z=\left\{(\sigma, w) \in \mathbf{Z},\left.w\right|_{\partial \Omega}=-\mathbf{v}_{\infty},\left.(\operatorname{div} w)\right|_{\partial \Omega}=0\right\}
$$

is a closed subset of $\mathbf{Z}$.

We sometimes write $\sigma \in \mathbf{Z}_{1}$ or $w \in \mathbf{Z}_{2}$; by this we mean precisely $(\sigma, 0) \in \mathbf{Z}$ or $(0, w) \in \mathbf{Z}$. Similarly, we use the short notation for the norms, e.g.,

$$
\|\sigma\|_{\mathbf{z}_{1}, \lambda}=\|(\sigma, 0)\|_{\mathbf{z}, \lambda}, \quad\|w\|_{\mathbf{z}_{2}, \lambda}=\|(0, w)\|_{\mathbf{z}, \lambda} .
$$

The set

$$
Z_{2}=\left\{w \in \mathbf{Z}_{2},\left.w\right|_{\partial \Omega}=-\mathbf{v}_{\infty},\left.(\operatorname{div} w)\right|_{\partial \Omega}=0\right\}
$$

is a closed subset of $\mathbf{Z}_{2}$. 
For any $w \in Z_{2}$ and $\mathbf{v}_{\infty} \in \mathbb{R}^{2}$ define

$$
\mathbf{W}_{w+\mathbf{v}_{\infty}}(\Omega)=\left\{\tau: \tau \in \mathbf{Z}_{1}, \operatorname{div}\left(\tau\left(w+\mathbf{v}_{\infty}\right)\right) \in \mathbf{Z}_{1}\right\} .
$$

This is a Banach space equipped with the norm

$$
\|\tau\|_{\mathbf{w}_{w+\mathbf{v}_{\infty}}}=\|\tau\|_{\mathbf{z}_{1, \lambda}}+\left\|\operatorname{div}\left(\tau\left(w+\mathbf{v}_{\infty}\right)\right)\right\|_{\mathbf{z}_{1, \lambda}}
$$

cf. Novotný and Padula [45].

Finally, denote, for $k=1, \ldots$ :

$$
\mathbf{L}=W^{1, q}(\Omega) \cap W^{k, p}(\Omega),
$$

the Banach space with the norm

$$
\|\cdot\|_{\mathbf{L}}=\|\cdot\|_{1, q}+\|\cdot\|_{k, p}
$$

and by

$$
\begin{aligned}
\mathbf{D}= & W^{2, q}(\Omega) \cap W^{k+1, p}(\Omega) \cap\left(\hat{H}_{\infty}^{1, p^{\prime}}(\Omega)\right)^{*} \\
& \cap\left(\hat{H}_{\infty}^{1,(3 q /(3-2 q))^{\prime}}(\Omega)\right)^{*} \cap\left\{g:\left.g\right|_{\partial \Omega}=0\right\}
\end{aligned}
$$

(as usual, $t^{\prime}$ denotes the dual exponent to $t: t^{\prime-1}+t^{-1}=1$ ) the Banach space with the norm

$$
\|\cdot\|_{\mathbf{D}}=|\cdot|_{*, p}+|\cdot|_{*, 3 q /(3-2 q)}+\|\cdot\|_{2, q}+\|\cdot\|_{k+1, p} \cdot
$$

The spaces of traces are denoted shortly

$$
\mathbf{B}=W^{k+2-1 / p, p}(\partial \Omega) \cap W^{3-1 / q, q}(\partial \Omega) \cap W^{1-(3-q) / 3 q, 3 q /(3-q)}(\partial \Omega)
$$

and equipped with the usual norm

$$
\|\cdot\|_{\mathbf{B}}=\|\cdot\|_{k+2-1 / p, p, \partial \Omega}+\|\cdot\|_{3-1 / q, q, \partial \Omega}+\|\cdot\|_{1-(3-q) / 3 q, 3 q /(3-q), \partial \Omega} .
$$

It is easily seen, that for $k \geq 1$ and $1<q<6 / 5, p>2$

$$
\begin{gathered}
\mathbf{B}=W^{k+2-1 / p, p}(\partial \Omega), \\
\|\cdot\|_{\mathbf{B}}=\|\cdot\|_{k+2-1 / p, p, \partial \Omega} .
\end{gathered}
$$

In the sequel, we adopt the following convention. If $\mathbf{Z},\left(\mathbf{Z}_{1}, \mathbf{Z}_{2}, Z, Z_{2}, \mathbf{L}\right.$, $\mathbf{B}, \mathbf{D})$ are the spaces (or the subsets) corresponding to an index $k(\geq 1$ ), then $\mathbf{Z}^{\prime}\left(\mathbf{Z}_{1}^{\prime}, \mathbf{Z}_{2}^{\prime}, Z^{\prime}, Z_{2}^{\prime}, \mathbf{L}^{\prime}, \mathbf{B}^{\prime}, \mathbf{D}^{\prime}\right)$ are the same spaces corresponding to the index $k-1$ for the $L^{p}$-summability and to one derivative less for the $L^{q}$-summability; e.g., if we take

$$
\mathbf{L}=W^{1, q}(\Omega) \cap W^{k, p}(\Omega),
$$


with the norm

$$
\|\cdot\|_{\mathbf{L}}=\|\cdot\|_{1, q}+\|\cdot\|_{k, p}
$$

then

$$
\mathbf{L}^{\prime}=L^{q}(\Omega) \cap W^{k-1, p}(\Omega)
$$

with the norm

$$
\|\cdot\|_{\mathbf{L}^{\prime}}=\|\cdot\|_{0, q}+\|\cdot\|_{k-1, p} .
$$

Now, we recall some more or less classical results about the functional spaces, which are adapted to our situation.

Lemma 4.1. Let $\Omega \in \mathcal{C}^{2}, 1<t<2<p<\infty, 1<s<\infty$.

i) Let $z \in L_{\text {loc }}^{t}(\Omega), \nabla z \in L^{t}(\Omega)$. Then there exists just one $z_{\infty} \in \mathbb{R}^{1}$ such that $z-z_{\infty} \in L^{2 t /(2-t)}(\Omega)$ and

$$
\begin{gathered}
\left\|z-z_{\infty}\right\|_{0,2 t /(2-t)} \leq c|z|_{1, t}, \\
\int_{\mathcal{S}}\left|z-z_{\infty}\right|^{t} d S \leq c|x|^{t-2}|z|_{1, t, B|x|}^{t}
\end{gathered}
$$

where $\mathcal{S}$ is a unit sphere and $d S$ is an infinitesimal element on it. If $z \in \hat{H}_{\infty}^{1, t}(\Omega)\left(\right.$ or $\left.z \in \hat{H}_{0}^{1, t}(\Omega)\right)$ then $z_{\infty}=0$ and

$$
\|z\|_{0,2 t /(2-t)} \leq c|z|_{1, t} .
$$

ii) The following characterisation holds true:

$$
\begin{gathered}
\hat{H}_{0}^{1, t}(\Omega)=\left\{z: z \in L^{t}{ }_{\mathrm{loc}}(\Omega), \nabla z \in L^{t}(\Omega), z_{\infty}=0,\left.z\right|_{\partial \Omega}=0\right\} \\
=\left\{z: z \in L^{2 t /(2-t)}(\Omega), \nabla z \in L^{t}(\Omega),\left.z\right|_{\partial \Omega}=0\right\}, \\
\hat{H}_{0}^{1, p}(\Omega)=\left\{z: z \in L^{p}{ }_{\mathrm{loc}}(\Omega), \nabla z \in L^{p}(\Omega),\left.z\right|_{\partial \Omega}=0\right\}, \\
\hat{H}_{\infty}^{1, t}(\Omega)=\left\{z: z \in L^{t}{ }_{\mathrm{loc}}(\Omega), \nabla z \in L^{t}(\Omega), z_{\infty}=0\right\} \\
=\left\{z: z \in L^{2 t /(2-t)}(\Omega), \nabla z \in L^{t}(\Omega)\right\}, \\
\hat{H}_{\infty}^{1, p}(\Omega)=\left.\left\{z: z \in L^{p}{ }_{\mathrm{loc}}(\Omega), \nabla z \in L^{p}(\Omega)\right\}\right|_{\mathbb{R}^{1}},
\end{gathered}
$$

where the symbol $\left.\right|_{\mathbb{R}^{1}}$ denotes the factorization with respect to the addition of a real constant.

iii) We have $L^{2 p /(2+p)}(\Omega) \subset \hat{H}^{-1, p}(\Omega)$ and

$$
|z|_{-1, p} \leq c\|z\|_{0,2 p /(2+p)}
$$


iv) Let $1<q<t<p<+\infty$. Then $L^{q}(\Omega) \cap L^{p}(\Omega) \subset L^{t}(\Omega)$ and

$$
\|z\|_{0, t}^{t} \leq c\|z\|_{0, q}^{(1-a) t}\|z\|_{0, p}^{a t}, \quad a=\frac{p(t-q)}{t(p-q)}
$$

In particular

$$
\|z\|_{0, t} \leq c\left(\|z\|_{0, q}+\|z\|_{0, p}\right) .
$$

v) We also have $W^{k, s}(\Omega) \subset \mathcal{C}^{l}(\bar{\Omega})$ for $(k-l) s>2$

$$
|z|_{\mathcal{C}^{l}} \leq c\|z\|_{k, s}
$$

Using Lemma 4.1 , we derive several properties of the spaces $\mathbf{Z}, \mathbf{Z}_{1}, \mathbf{Z}_{2}$, with respect to the norms $\|\cdot\|_{\mathbf{z}, \lambda},\|\cdot\|_{\mathbf{z}_{1}, \lambda},\|\cdot\|_{\mathbf{z}_{2, \lambda}}$.

Let $q \in(1,6 / 5)$ then $3 q /(3-q) \in(3 / 2,2), 3 q /(3-2 q) \in(3,6), 2 q /(2-q) \in$ $(2,3)$, and $6 q /(6-5 q) \in(6,+\infty)$. Suppose that $(\sigma, u) \in \mathbf{Z}$ with $q \in(1,6 / 5)$, and $p>2$. If $\nabla u \in W^{1, p}(\Omega)$ with $p>2$, we have (since $\nabla u \in L^{3 q /(3-q)}(\Omega)$ ), $\nabla u \in L^{s}(\Omega), s \in(3 q /(3-q), p)$, hence $u \in L^{s}(\Omega), s \in(6 q /(6-5 q), \infty)$. Moreover $\nabla^{2} u \in L^{q}(\Omega) \cap L^{p}(\Omega)$ and therefore $\nabla u \in L^{s}(\Omega), s \in(2 q /(2-$ $q), \infty)$. This implies

$$
|u|_{\mathcal{C}^{0}} \leq c \lambda^{-1 / 3}\|u\|_{\mathbf{z}_{2}, \lambda}
$$

For completeness, we recall the obvious imbeddings

$$
|\nabla u|_{\mathcal{C}^{k}} \leq c\|\nabla u\|_{k+1, p} \leq c \lambda^{-1 / 3}\|u\|_{\mathbf{Z}_{2}, \lambda}
$$

If $\nabla \sigma \in W^{1, q}(\Omega)$ then also $\nabla \sigma \in L^{2 q /(2-q)}$ and $\sigma \in L^{2 q /(2-q)}$; hence

$$
|\sigma|_{\mathcal{C}^{0}} \leq c \lambda^{-1 / 3}\|\sigma\|_{\mathbf{z}_{1}, \lambda}
$$

If $\nabla^{2} u \in L^{q}(\Omega) \cap L^{p}(\Omega)$ then $\nabla^{2} u \in L^{2}(\Omega)$ and

$$
\left\|\nabla^{2} u\right\|_{0,2} \leq c \lambda^{-1 / 3}\|u\|_{\mathbf{z}_{2}, \lambda} .
$$

If $\nabla u \in L^{3 q /(3-q)}(\Omega) \cap L^{p}(\Omega)$ then $\nabla u \in L^{2}(\Omega)$ and

$$
\|\nabla u\|_{0,2} \leq c \lambda^{-1 / 3}\|u\|_{\mathbf{z}_{2, \lambda}}
$$




\section{Main Theorems.}

The objective of this paper is to prove the following main theorem concerning existence for the full non linear system.

Theorem 5.1. Put $\lambda=\left|v_{\infty}\right|$. Let $k=1,2, \ldots, 1<q<6 / 5, p \geq \frac{3 q}{3-2 q}$, $\Omega \in \mathcal{C}^{k+3}$ and

$$
f \in \mathbf{L} \text {. }
$$

There exists a positive constant $\gamma_{0}$, dependent only of $k, q, p, \partial \Omega$ such that if

$$
0<\left|v_{\infty}\right| \leq \gamma_{0}
$$

then we have: There exist positive constants $\gamma_{1}$ and $\gamma_{2}$ depending of $k, q, p$, $\partial \Omega$ and $\lambda=\left|v_{\infty}\right|$ such that if

$$
\|f\|_{\mathbf{L}} \leq \gamma_{1}
$$

then the problem (2.3) admits just one solution $(\sigma, v)$ in the closed subset (of Z)

$$
B_{\gamma_{2}}(0)=\left\{(\sigma, v) \in Z \subset \mathbf{Z},\|(\sigma, v)\|_{\mathbf{z}, \lambda} \leq \gamma_{2}\right\} .
$$

This solution satisfies the estimate

$$
\|(\sigma, v)\|_{\mathbf{z}, \lambda} \leq c_{1}^{\prime}\left(\|f\|_{\mathbf{L}}+|\ln \lambda|^{-1} \lambda^{1+2(1-1 / q)}\right)
$$

The theorem below furnishes existence and estimates for the linearized system (2.4). The proof of Theorem 5.1 essentially relies on it.

Theorem 5.2. Put $\lambda=\left|v_{\infty}\right|$. Let $k=1,2, \ldots, 1<q<6 / 5,3 q /(3-2 q) \leq$ $p<+\infty, \Omega \in \mathcal{C}^{k+3}$ and

$$
w \in Z_{2} \subset \mathbf{Z}_{2}, \quad \mathcal{F} \in \mathbf{L}, \quad g \in \mathbf{D}, \quad \Psi \in \mathbf{B} .
$$

There exists a positive constant $\gamma$, dependent only of $k, q, p, \partial \Omega$, such that if

$$
\lambda^{-2 / 3}\|w\|_{\mathbf{z}_{2, \lambda}}+\lambda^{2 / 3} \leq \gamma
$$

then the problem (2.4) admits one and only one solution $(\sigma, v) \in Z \subset \mathbf{Z}$. Moreover, it satisfies the estimate

$$
\|(\sigma, v)\|_{\mathbf{Z}, \lambda} \leq c_{2}^{\prime}\left(\|\mathcal{F}\|_{\mathbf{L}}+\|g\|_{\mathbf{D}}+|\ln \lambda|^{-1} \lambda^{2(1-1 / q)}\|\Psi\|_{\mathbf{B}}\right) .
$$

Estimate (5.7) holds also with $\mathbf{Z}^{\prime}, \mathbf{L}^{\prime}, \mathbf{D}^{\prime}$, and $\mathbf{B}^{\prime}$ in place of $\mathbf{Z}, \mathbf{L}, \mathbf{D}$ and $\mathbf{B}$.

As we already pointed out in Section 4 , the constants $c_{1}^{\prime}, c_{2}^{\prime}$ are independent of $\lambda$. However, the estimates (5.4) and (5.7) are not uniform with respect to $\lambda$ in the sense that the norms $\|\cdot\|_{\mathbf{z}, \lambda}$ degenerate as $\lambda \rightarrow 0$ and $\gamma_{1}, \gamma_{2} \rightarrow 0$ as $\lambda \rightarrow 0$, cf. (8.10). 


\section{Preliminary Results.}

Consider in $\Omega$ the following Dirichlet problem

$$
\begin{gathered}
\Delta \theta=\varpi, \\
\left.\theta\right|_{\partial \Omega}=0, \\
\theta \rightarrow 0 \quad \text { as } \quad|x| \rightarrow+\infty .
\end{gathered}
$$

The theorem below is standard, see Simader and Sohr [53].

Lemma 6.1'. Let $\Omega \in \mathcal{C}^{2}$ and $\varpi \in \hat{H}^{-1,2}(\Omega)$. Then there exists one and only one weak solution of the problem (6.1), $\theta \in \hat{H}_{0}^{1,2}(\Omega)$, which satisfies the estimate

$$
|\theta|_{1,2} \leq c|\varpi|_{-1,2}
$$

For our purpose, we need some regularity results for the weak solutions to the equation (6.1).

Lemma 6.1. Let $1<t<\infty, k=0,1, \ldots, \Omega \in \mathcal{C}^{k+2}$ and

$$
\varpi \in \hat{H}^{-1,2}(\Omega) \cap W^{k, t}(\Omega) .
$$

There exists just one solution

$$
\theta \in \hat{H}_{0}^{1,2}(\Omega), \nabla^{2} \theta \in W^{k, t}(\Omega),
$$

of the problem (6.1) which satisfies the estimate

$$
\|\nabla \theta\|_{0,2}+\left\|\nabla^{2} \theta\right\|_{k, t} \leq c\left[|\varpi|_{-1,2}+\|\varpi\|_{k, t}\right] .
$$

Next, we consider in $\Omega$ the following Neumann problem

$$
\begin{gathered}
\Delta \varphi=\varpi, \\
\left.\frac{\partial \varphi}{\partial \nu}\right|_{\partial \Omega}=\psi, \\
\nabla \varphi \rightarrow 0 \quad \text { as } \quad|x| \rightarrow+\infty .
\end{gathered}
$$

The lemmas below are also standard, [54].

Lemma 6.2'. Let $1<t<+\infty, \Omega \in \mathcal{C}^{2}$ and $\varpi \in\left(\hat{H}_{\infty}^{1, t^{\prime}}(\Omega)\right)^{*}, \psi$ be such that the functional $\mathcal{G}$ defined by

$$
\langle\mathcal{G}, \eta\rangle=-\langle\varpi, \eta\rangle+\int_{\partial \Omega} \psi \eta d S, \quad \eta \in \hat{H}_{\infty}^{1, t^{\prime}}(\Omega)
$$


is a continuous linear functional on $\hat{H}_{\infty}^{1, t^{\prime}}(\Omega)\left(\right.$ i.e., $\left.\mathcal{G} \in\left(\hat{H}_{\infty}^{1, t^{\prime}}(\Omega)\right)^{*}\right)$. Then there exists just one weak solution $\varphi$ of the problem (6.4) which satisfies the estimate

$$
|\varphi|_{1, t} \leq c|\mathcal{G}|_{*, t}
$$

Remark 6.1. We notice that the conditions of Lemma $6.2^{\prime}$ are satisfied, e.g., when

$$
\varpi \in\left(\hat{H}_{\infty}^{1, t^{\prime}}(\Omega)\right)^{*}, \psi \in \mathcal{C}^{1}(\partial \Omega), \quad 2<t<\infty .
$$

Using the uniqueness arguments, we can prove

Lemma 6.2. Let $2<s, p<+\infty, 1<q<+\infty, k=0,1, \ldots, \Omega \in \mathcal{C}^{k+3}$ and

$$
\begin{gathered}
\varpi \in\left(\hat{H}_{\infty}^{1, s^{\prime}}(\Omega)\right)^{*} \cap\left(\hat{H}_{\infty}^{1, p^{\prime}}(\Omega)\right)^{*} \cap W^{2, q}(\Omega) \cap W^{k+1, p}(\Omega), \\
\psi \in W^{1-1 / s, s}(\partial \Omega) \cap W^{3-1 / q, q}(\partial \Omega) \cap W^{k+2-1 / p, p}(\partial \Omega) .
\end{gathered}
$$

Then there exists just one solution

$$
\varphi \in \hat{H}_{\infty}^{1, s}(\Omega) \cap \mathcal{H}_{\infty}^{k+3, p}(\Omega) \cap W_{\text {loc }}^{4, q}(\Omega), \nabla^{2} \varphi \in W^{2, q}(\Omega)
$$

of the problem (6.4), which satisfies the estimate

$$
\begin{gathered}
\|\nabla \varphi\|_{0, s}+\|\nabla \varphi\|_{k+2, p}+\left\|\nabla^{2} \varphi\right\|_{2, q} \\
\leq c\left[|\varpi|_{*, s}+|\varpi|_{*, p}+\|\varpi\|_{2, q}+\|\varpi\|_{k+1, p}\right. \\
\left.+\|\psi\|_{1-1 / s, s, \partial \Omega}+\|\psi\|_{3-1 / q, q, \partial \Omega}+\|\psi\|_{2+k-1 / p, p, \partial \Omega}\right] .
\end{gathered}
$$

Next, we shall investigate the following Oseen problem.

$$
\begin{gathered}
-\mu \Delta u+\nabla \Pi+\lambda \frac{\partial u}{\partial x_{1}}=\varpi, \\
\operatorname{div} u=h, \\
\left.u\right|_{\partial \Omega}=\psi, \\
u(x) \rightarrow 0, \Pi(x) \rightarrow 0 \text { as }|x| \rightarrow+\infty .
\end{gathered}
$$

A wide analysis for a study of the above problems has been developed in Galdi [24]; in particular, we recall the following results, see [24] Vol. II, Ch. 10 or $[26]$ : 
Lemma 6.3. Let $\lambda_{0}>0, \lambda \in\left(0, \lambda_{0}\right), 1<q<6 / 5, \Omega \in \mathcal{C}^{2}$ and $\varpi \in L^{q}(\Omega)$, $h \in W^{1, q}(\Omega), \psi \in W^{2-1 / q, q}(\partial \Omega)$. Then there exists just one solution $(u, \Pi)$

$$
\begin{gathered}
u \in L^{3 q /(3-2 q)}(\Omega) \cap \hat{H}_{\infty}^{1,3 q /(3-q)}(\Omega), \nabla^{2} u \in L^{q}(\Omega), \nabla u_{2}, \frac{\partial u}{\partial x_{1}} \in L^{q}(\Omega), \\
u_{2} \in L^{2 q /(2-q)}(\Omega), \Pi \in L^{2 q /(2-q)}(\Omega), \quad \nabla \Pi \in L^{q}(\Omega)
\end{gathered}
$$

to the problem (6.8) which satisfies the estimate

$$
\begin{gathered}
\lambda^{2 / 3}\|u\|_{0,3 q /(3-2 q)}+\lambda\left(\left\|\frac{\partial u}{\partial x_{1}}\right\|_{0, q}+\left\|u_{2}\right\|_{0,2 q /(2-q)}+\left\|\nabla u_{2}\right\|_{0, q}\right) \\
+\lambda^{1 / 3}\left(\|\nabla u\|_{0,3 q /(3-q)}+\left\|\nabla^{2} u\right\|_{0, q}+\|\nabla \Pi\|_{0, q}+\|\Pi\|_{0,2 q /(2-q)}\right) \\
\leq c\left[\|\varpi\|_{0, q}+\|h\|_{1, q}+|\ln \lambda|^{-1} \lambda^{2(1-1 / q)}\|\psi\|_{2-1 / q, q, \partial \Omega}\right] .
\end{gathered}
$$

The positive constant $c$ in the estimate (6.10) is independent of $\lambda$; however, it possibly depends of $\lambda_{0}$.

We also easily verify, see Appendix, the following statement about the regularity:

Lemma 6.4. Let $\lambda_{0}>0, \lambda \in\left(0, \lambda_{0}\right), 1<q<6 / 5, \frac{2 q}{2-q} \leq p<+\infty$, $k=0,1, \ldots, \Omega \in \mathcal{C}^{k_{0}}, k_{0}=\max \{3, k+2\}$ and

$$
\begin{gathered}
h \in W^{k+1, p}(\Omega) \cap W^{2, q}(\Omega), \varpi \in W^{k, p}(\Omega) \cap W^{1, q}(\Omega), \\
\psi \in W^{k+2-1 / p, p}(\partial \Omega) \cap W^{3-1 / q, q}(\partial \Omega) .
\end{gathered}
$$

Then there exists just one solution $(u, \Pi)$

$$
\begin{gathered}
u \in \mathcal{H}_{\infty}^{k+2, p}(\Omega) \cap L^{3 q /(3-2 q)}(\Omega) \cap \hat{H}_{\infty}^{1,3 q /(3-q)}(\Omega), \\
\nabla^{2} u \in W^{1, q}(\Omega), \nabla u_{2}, \frac{\partial u}{\partial x_{1}} \in L^{q}(\Omega), u_{2} \in L^{2 q /(2-q)}(\Omega), \\
\Pi \in \mathrm{E}^{2 q /(2-q)}(\Omega) \cap W^{k+1, p}(\Omega), \nabla \Pi \in W^{1, q}(\Omega),
\end{gathered}
$$


to the problem (6.8) which satisfies the estimate

$$
\begin{gathered}
\lambda^{2 / 3}\|u\|_{0,3 q /(3-2 q)}+\lambda\left(\left\|\frac{\partial u}{\partial x_{1}}\right\|_{0, q}+\left\|u_{2}\right\|_{0,2 q /(2-q)}+\left\|\nabla u_{2}\right\|_{0, q}\right) \\
+\lambda^{1 / 3}\left(\|\nabla u\|_{0,3 q /(3-q)}+\left\|\nabla^{2} u\right\|_{1, q}+\|\nabla u\|_{k+1, p}\right. \\
\left.+\|\nabla \Pi\|_{1, q}+\|\Pi\|_{0,2 q /(2-q)}+\|\Pi\|_{k+1, p}\right) \\
\leq c\left[\|\varpi\|_{1, q}+\|\varpi\|_{k, p}+\|h\|_{2, q}+\|h\|_{k+1, p}\right. \\
\left.+|\ln \lambda|^{-1} \lambda^{2(1-1 / q)}\left(\|\psi\|_{3-1 / q, q, \partial \Omega}+\|\psi\|_{k+2-1 / p, p, \partial \Omega}\right)\right] .
\end{gathered}
$$

The positive constant $c$ is independent of $\lambda$; however, as in the previous lemma, it possibly depends of $\lambda_{0}$.

Finally, we consider the steady transport equation

$$
\omega+\operatorname{div}\left(\left(w+\mathbf{v}_{\infty}\right) \omega\right)=\varpi,
$$

with the condition

$$
\left.\left(\left(w+\mathbf{v}_{\infty}\right) \cdot \nu\right)\right|_{\partial \Omega}=0 .
$$

For the proof of the following statement see Theorem 6.3 in [42].

Lemma 6.5. Let $1<q<2, p>2, l, k=1,2 \ldots, l \leq k, \Omega \in \mathcal{C}^{k}, \mathbf{v}_{\infty} \in \mathbb{R}^{2}$ and

$$
\begin{gathered}
w \in \mathcal{H}_{\infty}^{k+2, p}(\Omega), \nabla^{2} w \in W^{l, q}(\Omega),\left.\left(\left(w+\mathbf{v}_{\infty}\right) \cdot \nu\right)\right|_{\partial \Omega}=0, \\
\nabla \varpi \in W^{l, q}(\Omega), \varpi \in W^{k+1, p}(\Omega) .
\end{gathered}
$$

There exists $\gamma>0$ such that if

$$
\vartheta_{0}=\left\|\nabla^{2} w\right\|_{l, q}+\|\nabla w\|_{k+1, p}+\left|\mathbf{v}_{\infty}\right| \leq \gamma
$$

then the problem (6.13) admits just one solution $\omega$

$$
\begin{gathered}
\omega \in W^{k+1, p}(\Omega), \nabla \omega \in W^{l, q}(\Omega), \\
\operatorname{div}\left(\left(w+\mathbf{v}_{\infty}\right) \omega\right) \in W^{k+1, p}(\Omega), \nabla \operatorname{div}\left(\left(w+\mathbf{v}_{\infty}\right) \omega\right) \in W^{l, q}(\Omega),
\end{gathered}
$$

satisfying the estimate

$$
\begin{gathered}
\|\nabla \omega\|_{l, q}+\|\omega\|_{k+1, p}+\left\|\nabla \operatorname{div}\left(\left(w+\mathbf{v}_{\infty}\right) \omega\right)\right\|_{l, q} \\
+\left\|\operatorname{div}\left(\left(w+\mathbf{v}_{\infty}\right) \omega\right)\right\|_{k+1, p} \leq c\left(\|\nabla \varpi\|_{l, q}+\|\varpi\|_{k+1, p}\right) .
\end{gathered}
$$

Moreover we have

$$
\Delta \omega \in W^{k-1, p}(\Omega) \cap W^{l-1, q}(\Omega),
$$


and

$$
\begin{gathered}
\|\Delta \omega\|_{l-1, q}+\|\Delta \omega\|_{k-1, p} \\
+\left\|\Delta \operatorname{div}\left(\left(w+\mathbf{v}_{\infty}\right) \omega\right)\right\|_{l-1, q}+\left\|\Delta \operatorname{div}\left(\left(w+\mathbf{v}_{\infty}\right) \omega\right)\right\|_{k-1, p} \\
\leq c\left\{\|\Delta \varpi\|_{k-1, p}+\|\Delta \varpi\|_{l-1, q}+\vartheta_{0}\left(\|\omega\|_{k+1, p}+\|\nabla \omega\|_{l, q}\right)\right\} .
\end{gathered}
$$

\section{Proof of Theorem 5.2.}

We prove that the operator $\mathcal{L}$ formally defined by (3.3) (see (3.4)-(3.6)) has the following properties:

(i) If $w \in Z_{2} \subset \mathbf{Z}_{2}$, and $\lambda$ and $\|w\|_{\mathbf{z}_{2}, \lambda}$ are sufficiently small then it is defined on $\mathbf{W}_{w+\mathbf{v}_{\infty}}(\Omega)$ (provided $\mathcal{F} \in \mathbf{L}, g \in \mathbf{D}$ and $\Psi \in \mathbf{B}$ ).

(ii) It is a contraction on $\mathbf{W}_{w+\mathbf{v}_{\infty}}(\Omega)$ and therefore there exists a unique fixed point $\sigma \in \mathbf{W}_{w+\mathbf{v}_{\infty}}(\Omega)$.

(iii) The couple $(\sigma, v)$ with $\sigma$ being the fixed point of $\mathcal{L}, \sigma \in \mathbf{W}_{w+\mathbf{v}_{\infty}}(\Omega) \subset$ $\mathbf{Z}_{1}$ and $v=u+\nabla \varphi \in \mathbf{Z}_{2}$, where $\varphi$ is a (unique) solution of the problem (3.4) (i.e. $\nabla \varphi \in \mathbf{Z}_{2}$ ) corresponding to $\tau=\sigma$ and $u \in \mathbf{Z}_{2}$ is a (unique) solution of the problem (3.5), satisfies system (2.4) and the estimate (5.7).

Recall that for $1<q<6 / 5,3 q /(3-q) \in(3 / 2,2), 3 q /(3-2 q) \in(3,6)$, $2 q /(2-q) \in(2,3)$ and $6 q /(6-5 q) \in(6,+\infty)$. Put $\lambda=\left|v_{\infty}\right|$. Lemma 6.2 applied to problem (3.4) gives

$$
\begin{gathered}
\|\nabla \varphi\|_{0,3 q /(3-2 q)}+\left\|\nabla^{2} \varphi\right\|_{2, q}+\|\nabla \varphi\|_{k+2, p} \\
\leq c\left\{\|g\|_{\mathbf{D}}+\left|\operatorname{div}\left(\tau\left(w+\mathbf{v}_{\infty}\right)\right)\right|_{*, 3 q /(3-2 q)}+\left|\operatorname{div}\left(\tau\left(w+\mathbf{v}_{\infty}\right)\right)\right|_{*, p}\right. \\
\left.+\left\|\operatorname{div}\left(\tau\left(w+\mathbf{v}_{\infty}\right)\right)\right\|_{2, q}+\left\|\operatorname{div}\left(\tau\left(w+\mathbf{v}_{\infty}\right)\right)\right\|_{k+1, p}+\|\Psi\|_{\mathbf{B}}\right\}
\end{gathered}
$$

Here we list some important consequences of the Sobolev inequality and the interpolation arguments:

(i) Since $\nabla^{3} \varphi \in L^{q}(\Omega), \nabla^{2} \varphi \in L^{2 q /(2-q)}(\Omega)$, thus $\nabla^{2} \varphi \in L^{s}(\Omega), s \in\left[q, \frac{2 q}{2-q}\right]$, this implies $\nabla^{2} \varphi \in L^{3 q /(3-q)}(\Omega)$, actually, this follows from the following inequalities

$$
q<\frac{3 q}{3-q}<\frac{2 q}{2-q}, \quad\left\|\nabla^{2} \varphi\right\|_{0,3 q /(3-q)} \leq c\left\|\nabla^{2} \varphi\right\|_{1, q} .
$$

(ii) The inequality below is the well known Sobolev inequality

$$
\left\|\nabla^{2} \varphi\right\|_{0,2 q /(2-q)} \leq c\left\|\nabla^{2} \varphi\right\|_{1, q} .
$$


(iii) By using the imbedding theorems for Sobolev spaces and (4.1)-(4.5), we deduce the following estimates

$$
\begin{aligned}
\left|\operatorname{div}\left(\tau\left(w+\mathbf{v}_{\infty}\right)\right)\right|_{*, 3 q /(3-2 q)} & \leq\left\|\tau\left(w+\mathbf{v}_{\infty}\right)\right\|_{0,3 q /(3-2 q)} \\
& \leq c\|\tau\|_{0,3 q /(3-2 q)}\left(|w|_{\mathcal{C}^{0}}+\lambda\right) \\
& \leq c\left(\lambda^{-2 / 3}\|w\|_{\mathbf{z}_{2}, \lambda}+\lambda^{2 / 3}\right)\|\tau\|_{\mathbf{z}_{1}, \lambda}
\end{aligned}
$$

since $\frac{2 q}{2-q}<\frac{3 q}{3-2 q} \leq p$ and $\|\tau\|_{0,3 q /(3-2 q)} \leq c \lambda^{-1 / 3}\|\tau\|_{\mathbf{Z}_{1}, \lambda} ;$

$$
\begin{aligned}
\left\|\operatorname{div}\left(\tau\left(w+\mathbf{v}_{\infty}\right)\right)\right\|_{1, q} \leq & \left\|\nabla \tau \cdot\left(w+\mathbf{v}_{\infty}\right)\right\|_{1, q}+\|\tau \operatorname{div} w\|_{1, q} \\
\leq & c\left(\|\nabla \tau\|_{1, q}\left(|w|_{\mathcal{C}^{0}}+\lambda\right)+\|\nabla \tau\|_{0, q}|\nabla w|_{\mathcal{C}^{0}}\right. \\
& \left.+\|\tau\|_{0,2 q /(2-q)}\left\|\nabla^{2} w\right\|_{0,2}\right) \\
\leq & c\left(\lambda^{-2 / 3}\|w\|_{\mathbf{z}_{2, \lambda}}+\lambda^{2 / 3}\right)\|\tau\|_{\mathbf{z}_{1, \lambda}}
\end{aligned}
$$

since $\frac{3 q}{3-q}<2<p$ and therefore $\left\|\nabla^{2} w\right\|_{0,2} \leq c \lambda^{-1 / 3}\|w\|_{\mathbf{z}_{2}, \lambda}$,

$$
\begin{aligned}
\left|\operatorname{div}\left(\tau\left(w+\mathbf{v}_{\infty}\right)\right)\right|_{*, p} & \leq\left\|\tau\left(w+\mathbf{v}_{\infty}\right)\right\|_{0, p} \\
& \leq c\left(\lambda^{-2 / 3}\|w\|_{\mathbf{z}_{2}, \lambda}+\lambda^{2 / 3}\right)\|\tau\|_{\mathbf{z}_{1}, \lambda} \\
\left\|\operatorname{div}\left(\tau\left(w+\mathbf{v}_{\infty}\right)\right)\right\|_{k, p} & \leq\left\|\nabla \tau \cdot\left(w+\mathbf{v}_{\infty}\right)\right\|_{k, p}+\|\tau \operatorname{div} w\|_{k, p} \\
& \leq c\left(\lambda^{-2 / 3}\|w\|_{\mathbf{z}_{2, \lambda}}+\lambda^{2 / 3}\right)\|\tau\|_{\mathbf{z}_{1, \lambda}} .
\end{aligned}
$$

(iv) By Lemma 6.1, noticing that $\left.\left(w+\mathbf{v}_{\infty}\right)\right|_{\partial \Omega}=0$ and $\left.(\operatorname{div} w)\right|_{\partial \Omega}=0$, imply $\left.\left(\operatorname{div}\left(\tau\left(w+\mathbf{v}_{\infty}\right)\right)\right)\right|_{\partial \Omega}=0$, we obtain

$$
\begin{aligned}
\left\|\nabla^{2} \operatorname{div}\left(\tau\left(w+\mathbf{v}_{\infty}\right)\right)\right\|_{0, q} \leq & c\left\{\left\|\Delta \operatorname{div}\left(\tau\left(w+\mathbf{v}_{\infty}\right)\right)\right\|_{0, q}\right. \\
& \left.+\left|\Delta \operatorname{div}\left(\tau\left(w+\mathbf{v}_{\infty}\right)\right)\right|_{-1,2}\right\} \\
\left\|\nabla^{2} \operatorname{div}\left(\tau\left(w+\mathbf{v}_{\infty}\right)\right)\right\|_{k-1, p} \leq & c\left\{\left\|\Delta \operatorname{div}\left(\tau\left(w+\mathbf{v}_{\infty}\right)\right)\right\|_{k-1, p}\right. \\
& \left.+\left|\Delta \operatorname{div}\left(\tau\left(w+\mathbf{v}_{\infty}\right)\right)\right|_{-1,2}\right\} .
\end{aligned}
$$

(v) Finally, we have the estimate

$$
\begin{gathered}
\left|\Delta \operatorname{div}\left(\tau\left(w+\mathbf{v}_{\infty}\right)\right)\right|_{-1,2} \leq\left\|\nabla \tau\left(w+\mathbf{v}_{\infty}\right)\right\|_{1,2}+\|\tau \nabla \cdot w\|_{1,2} \\
\leq c\left(\lambda^{-2 / 3}\|w\|_{\mathbf{z}_{2}, \lambda}+\lambda^{2 / 3}\right)\|\tau\|_{\mathbf{z}_{1}, \lambda}
\end{gathered}
$$


Estimate (7.1) together with (7.2)-(7.8) yields

$$
\begin{gathered}
\|\nabla \varphi\|_{0,3 q /(3-2 q)}+\left\|\nabla^{2} \varphi\right\|_{2, q}+\left\|\nabla^{2} \varphi\right\|_{0,3 q /(3-q)}+\left\|\nabla^{2} \varphi\right\|_{0,2 q /(2-q)} \\
+\|\nabla \varphi\|_{k+2, p} \leq c\left\{\|g\|_{\mathbf{D}}+|\tau|_{w+\mathbf{v}_{\infty}, k-1, q, p}\right. \\
\left.+\left(\lambda^{-2 / 3}\|w\|_{\mathbf{Z}_{2}, \lambda}+\lambda^{2 / 3}\right)\|\tau\|_{\mathbf{z}_{1, \lambda}}+\|\Psi\|_{\mathbf{B}}\right\}
\end{gathered}
$$

Here, we have denoted, to simplify the notation

$$
|\tau|_{w+\mathbf{v}_{\infty}, k-1, q, p}=\left\|\Delta \operatorname{div}\left(\tau\left(w+\mathbf{v}_{\infty}\right)\right)\right\|_{0, q}+\left\|\Delta \operatorname{div}\left(\tau\left(w+\mathbf{v}_{\infty}\right)\right)\right\|_{k-1, p} .
$$

We find from the definition of the traces

$$
\|\nabla \varphi\|_{l-1 / s, s, \partial \Omega}=\|\nabla \varphi\|_{0, s, \partial \Omega}+\left\|\nabla^{2} \varphi\right\|_{l-1-1 / s, s, \partial \Omega},
$$

$l=2,3, \ldots, 1<s<+\infty$. If $s=q$, since $q<p$ and $\partial \Omega$ is compact, we get

$$
\|\nabla \varphi\|_{0, q, \partial \Omega} \leq c\|\nabla \varphi\|_{0, p, \partial \Omega} \leq c^{\prime}\|\nabla \varphi\|_{1-1 / p, p, \partial \Omega} .
$$

Employing the Gagliardo theorem about the traces, we therefore get

$$
\|\nabla \varphi\|_{3-1 / q, q, \partial \Omega} \leq c\left\{\|\nabla \varphi\|_{1, p}+\left\|\nabla^{2} \varphi\right\|_{2, q}\right\}
$$

and

$$
\|\nabla \varphi\|_{k+2-1 / p, p, \partial \Omega} \leq c\|\nabla \varphi\|_{k+2, p} .
$$

Now, we can apply Lemma 6.4 to the equation (3.5). To estimating the boundary integrals, we use the inequalities (7.11), (7.12), and consequently estimate (7.9), and thus get (recall that $\lambda^{1 / 3} \leq|\ln \lambda|^{-1} \lambda^{2(1-1 / q)}<1$, for $\lambda$ sufficiently small and $q \in(1,6 / 5))$ :

$$
\begin{gathered}
\|(\Pi, u)\|_{\mathbf{z}, \lambda} \leq c\left\{\|\mathcal{F}\|_{1, q}+\|\mathcal{F}\|_{k, p}+\|g\|_{\mathbf{D}}\right. \\
+|\ln \lambda|^{-1} \lambda^{2(1-1 / q)}\left[\left(\lambda^{-2 / 3}\|w\|_{\mathbf{z}_{2, \lambda}}+\lambda^{2 / 3}\right)\|\tau\|_{\mathbf{z}_{1, \lambda}}\right. \\
\left.\left.+\|\Psi\|_{\mathbf{B}}+|\tau|_{w+\mathbf{v}_{\infty}, k-1, q, p}\right]\right\} .
\end{gathered}
$$

Taking div of (3.5), one gets

$$
\Delta \Pi=\operatorname{div} \mathcal{F}
$$

which yields estimates

$$
\|\Delta \Pi\|_{0, q} \leq c\|\mathcal{F}\|_{1, q}, \quad\|\Delta \Pi\|_{k-1, p} \leq c\|\mathcal{F}\|_{k, p} .
$$


Applying Lemma 6.5 (estimate (6.14)) to the problem (3.6) and taking into account (7.13), one obtains

$$
\begin{gathered}
\|\sigma\|_{\mathbf{z}_{1, \lambda}}+\left\|\operatorname{div}\left(\sigma\left(w+\mathbf{v}_{\infty}\right)\right)\right\|_{\mathbf{z}_{1, \lambda}} \leq c\left\{\|\mathcal{F}\|_{1, q}+\right. \\
+\|\mathcal{F}\|_{k, p}+\|g\|_{\mathbf{D}}+|\ln \lambda|^{-1} \lambda^{2(1-1 / q)}\left[\left(\lambda^{-2 / 3}\|w\|_{\mathbf{z}_{2, \lambda}}+\lambda^{2 / 3}\right)\|\tau\|_{\mathbf{z}_{1}, \lambda}\right. \\
\left.\left.+\|\Psi\|_{\mathbf{B}}+|\tau|_{w+\mathbf{v}_{\infty}, k-1, q, p}\right]\right\} .
\end{gathered}
$$

Moreover, again by Lemma 6.5 (estimate (6.15)) and in virtue of (7.14), one gets

$$
\begin{gathered}
|\sigma|_{w+\mathbf{v}_{\infty}, k-1, q, p} \leq c\left\{\|\mathcal{F}\|_{1, q}+\|\mathcal{F}\|_{k, p}\right. \\
\left.\|g\|_{\mathbf{D}}+\left(\lambda^{-2 / 3}\|w\|_{\mathbf{z}_{2, \lambda}}+\lambda^{2 / 3}\right)\|\tau\|_{\mathbf{z}_{1, \lambda}}\right\}
\end{gathered}
$$

Estimates (7.15), (7.16) imply

$$
\begin{aligned}
& \|\sigma\|_{\mathbf{z}_{1}, \lambda}+\left\|\operatorname{div}\left(\sigma\left(w+\mathbf{v}_{\infty}\right)\right)\right\|_{\mathbf{z}_{1}, \lambda}+|\sigma|_{w+\mathbf{v}_{\infty}, k-1, q, p} \\
& \leq c_{1}\left\{\|\mathcal{F}\|_{\mathbf{L}}+\|g\|_{\mathbf{D}}+\left(\lambda^{-2 / 3}\|w\|_{\mathbf{z}_{2}, \lambda}+\lambda^{2 / 3}\right)\|\tau\|_{\mathbf{z}_{1}, \lambda}\right. \\
& \left.\quad+|\ln \lambda|^{-1} \lambda^{2(1-1 / q)}\left[\|\Psi\|_{\mathbf{B}}+|\tau|_{w+\mathbf{v}_{\infty}, k-1, q, p}\right]\right\} .
\end{aligned}
$$

For any fixed $\lambda>0$, the norms

$$
\begin{gathered}
\|\sigma\|_{\mathbf{W}_{w+\mathbf{v}_{\infty}}}=\|\sigma\|_{\mathbf{z}_{1, \lambda}}+\left\|\operatorname{div}\left(\sigma\left(w+\mathbf{v}_{\infty}\right)\right)\right\|_{\mathbf{z}_{1, \lambda},} \\
\|\sigma\|_{\mathbf{W}_{w+\mathbf{v}_{\infty}}}^{\prime}=\|\sigma\|_{\mathbf{z}_{1, \lambda}}+\left\|\operatorname{div}\left(\sigma\left(w+\mathbf{v}_{\infty}\right)\right)\right\|_{\mathbf{z}_{1, \lambda}}+|\sigma|_{w+\mathbf{v}_{\infty}, k-1, q, p}
\end{gathered}
$$

are equivalent in $\mathbf{W}_{w+\mathbf{v}_{\infty}}(\Omega)$. Therefore, the last inequality yields

$$
\begin{gathered}
\|\sigma\|_{\mathbf{W}_{w+\mathbf{v}_{\infty}}}^{\prime} \leq c_{2}\left\{\|\mathcal{F}\|_{\mathbf{L}}\right. \\
+\max \left[\left(\lambda^{-2 / 3}\|w\|_{\mathbf{z}_{2, \lambda}}+\lambda^{2 / 3}\right),|\ln \lambda|^{-1} \lambda^{2\left(1-\frac{1}{q}\right)}\right]\|\tau\|_{\mathbf{W}_{w+\mathbf{v}_{\infty}}}^{\prime} \\
\left.+\|g\|_{\mathbf{D}}+|\ln \lambda|^{-1} \lambda^{2(1-1 / q)}\|\Psi\|_{\mathbf{B}}\right\}
\end{gathered}
$$

(recall that $c_{2}>0$ is independent of $\lambda$ ). This furnishes the contraction and existence of a fixed point $\sigma \in \mathbf{W}_{w+\mathbf{v}_{\infty}}(\Omega)$ (recall that the operator $\mathcal{L}$ is linear), provided

$$
c_{2} \max \left[\left(\lambda^{-2 / 3}\|w\|_{\mathbf{z}_{2}, \lambda}+\lambda^{2 / 3}\right),|\ln \lambda|^{-1} \lambda^{2\left(1-\frac{1}{q}\right)}\right]<1,
$$

i.e. provided

$$
\max \left[\left(\lambda^{-2 / 3}\|w\|_{\mathbf{z}_{2}, \lambda}+\lambda^{2 / 3}\right),|\ln \lambda|^{-1} \lambda^{2\left(1-\frac{1}{q}\right)}\right]<\bar{\gamma}(<1) .
$$


The last condition implies the condition (5.6). It suffices to take $\gamma=$ $\min (\bar{\gamma}, \bar{\lambda})$, where $\bar{\lambda} \in(0,1)$ is a solution of the equation $|\ln \lambda|^{-1} \lambda^{2(1-1 / q)}$ $=\bar{\gamma}$.

Now, we are in a position to prove estimate (5.7). Estimate (7.17) written in the fixed point $\tau=\sigma$ yields, for $\gamma$ sufficiently small,

$$
\|\sigma\|_{\mathbf{z}_{1}, \lambda} \leq c\left\{\|\mathcal{F}\|_{\mathbf{L}}+\|g\|_{\mathbf{D}}+|\ln \lambda|^{-1} \lambda^{2(1-1 / q)}\|\Psi\|_{\mathbf{B}}\right\}
$$

The estimate (7.19) and the estimates (7.9), (7.13), written in the fixed point $\sigma$, and in the corresponding $v=u+\nabla \varphi$, give, for $\gamma$ sufficiently small,

$$
\|v\|_{\mathbf{Z}_{2}, \lambda} \leq c\left\{\|\mathcal{F}\|_{\mathbf{L}}+\|g\|_{\mathbf{D}}+|\ln \lambda|^{-1} \lambda^{2(1-1 / q)}\|\Psi\|_{\mathbf{B}}\right\}
$$

The last two estimates yield the inequality (5.7). Estimate (5.7) with $\mathbf{Z}^{\prime}, \mathbf{L}^{\prime}$, $\mathbf{D}^{\prime}, \mathbf{B}^{\prime}$ can be proved similarly. Theorem 5.2 is thus proved.

\section{Proof of Theorem 5.1}

The lines of the proof of Theorem 5.1 are the following:

(i) The map $\mathcal{T}$ (see (3.1)) is, for a fixed $\mathbf{v}_{\infty}\left(0<\left|v_{\infty}\right|<\gamma_{0}\right)$ and any $f \in \mathbf{L}$ $\left(\|f\|_{\mathbf{L}} \leq \gamma_{1}\right)$, well defined in a certain closed (convex) subset $B_{\gamma_{2}}(0)$ of $\mathbf{Z}$, provided $\gamma_{0}$ and $\gamma_{1}\left(\left|v_{\infty}\right|\right), \gamma_{2}\left(\left|v_{\infty}\right|\right)$ are sufficiently small, and maps $B_{\gamma_{2}}(0)$ into itself.

(ii) It is a contraction in $B_{\gamma_{2}}(0)$, in the topology of $\mathbf{Z}^{\prime}$. (For the definition of $\mathbf{Z}^{\prime}$ see Section 4.)

Once we prove these two assertions, the existence of a (unique) fixed point follows from a general version of the Banach contraction principle (cf. e.g., Zeidler [58]).

Let us start with the estimates of the nonlinear terms.

Lemma 8.1. Let $\lambda \in(0,1), 1<q<6 / 5, k=1, \ldots$, and $p>2$. Assume

$$
w \in \mathbf{Z}_{2}, \quad v \in \mathbf{Z}_{2}, \quad \sigma \in \mathbf{Z}_{1}, \quad f \in W^{1, q}(\Omega) .
$$


Then

$(8.1)$

$$
\begin{aligned}
& \|(1+\sigma) v \cdot \nabla w\|_{1, q} \leq c \lambda^{-1-2(1-1 / q)}\left(1+\lambda^{-1 / 3}\|\sigma\|_{\mathbf{z}_{1}, \lambda}\right)\|w\|_{\mathbf{z}_{2, \lambda}}\|v\|_{\mathbf{z}_{2, \lambda},}, \\
& \|(1+\sigma) v \operatorname{div} w\|_{1, q} \leq c \lambda^{-1-2(1-1 / q)}\left(1+\lambda^{-1 / 3}\|\sigma\|_{\mathbf{z}_{1, \lambda}}\right)\|w\|_{\mathbf{Z}_{2, \lambda}}\|v\|_{\mathbf{z}_{2, \lambda}}, \\
& \|\nabla \sigma w \otimes v\|_{1, q} \leq c \lambda^{-1-2(1-1 / q)}\|w\|_{\mathbf{Z}_{2, \lambda}}\|v\|_{\mathbf{z}_{2, \lambda}}\|\sigma\|_{\mathbf{Z}_{1, \lambda},}, \\
& \lambda\left\|\frac{\partial(\sigma v)}{\partial x_{1}}\right\|_{1, q} \leq c \lambda^{-1-2(1-1 / q)}\|\sigma\|_{\mathbf{z}_{1}, \lambda}\|v\|_{\mathbf{z}_{2}, \lambda} \\
& \|(1+\sigma) f\|_{0, q} \leq\|f\|_{0, q}+\lambda^{-1 / 3}\|\sigma\|_{\mathbf{z}_{1, \lambda}}\|f\|_{0, q}, \\
& \|\nabla((1+\sigma) f)\|_{0, q} \leq\|\nabla f\|_{0, q}+\lambda^{-1 / 3}\|\sigma\|_{\mathbf{z}_{1, \lambda} \lambda}\|f\|_{1, q} .
\end{aligned}
$$

Proof. We use, without pointing it out, the Hölder inequality, interpolation, and imbedding theorems, especially (4.1)-(4.5) (for all these tools see Section $4)$.

Proof of the estimates $(8.1)_{1-3}$. Put

$$
\begin{aligned}
& (1+\sigma) v \cdot \nabla w=\left(V_{1}^{(0)}, V_{2}^{(0)}\right), \\
& (1+\sigma) v \operatorname{div} w=\left(V_{1}^{(1)}, V_{2}^{(1)}\right) ;
\end{aligned}
$$

i.e.

$$
\begin{aligned}
V_{1}^{(0)} & =(1+\sigma) v_{1} \frac{\partial w_{1}}{\partial x_{1}}+(1+\sigma) v_{2} \frac{\partial w_{1}}{\partial x_{2}} \\
V_{2}^{(0)} & =(1+\sigma) v_{1} \frac{\partial w_{2}}{\partial x_{1}}+(1+\sigma) v_{2} \frac{\partial w_{2}}{\partial x_{2}} \\
V_{1}^{(1)} & =(1+\sigma) v_{1} \frac{\partial w_{1}}{\partial x_{1}}+(1+\sigma) v_{1} \frac{\partial w_{2}}{\partial x_{2}} \\
V_{2}^{(1)} & =(1+\sigma) v_{2} \frac{\partial w_{1}}{\partial x_{1}}+(1+\sigma) v_{2} \frac{\partial w_{2}}{\partial x_{2}} .
\end{aligned}
$$

Hence

$$
\begin{aligned}
\|(1+\sigma) v \cdot \nabla w\|_{0, q} \leq & \left(1+|\sigma|_{\mathcal{C}^{0}}\right)\left\{\left\|v_{1}\right\|_{0,3 q /(3-2 q)}\left\|\frac{\partial w}{\partial x_{1}}\right\|_{0,3 / 2}\right. \\
& \left.+\|\nabla w\|_{0,3 q /(3-q)}\left\|v_{2}\right\|_{0,3}\right\}
\end{aligned}
$$




$$
\begin{aligned}
\|(1+\sigma) v \nabla \cdot w\|_{0, q} \leq & \left(1+|\sigma|_{\mathcal{C}^{0}}\right)\left\{\|\nabla w\|_{0,3 q /(3-q)}\left\|v_{2}\right\|_{0,3}\right. \\
& \left.+\left\|v_{1}\right\|_{0,3 q /(3-2 q)}\left(\left\|\frac{\partial w_{1}}{\partial x_{1}}\right\|_{0,3 / 2}+\left\|\nabla w_{2}\right\|_{0,3 / 2}\right)\right\} .
\end{aligned}
$$

We have by the interpolation

$$
\begin{gathered}
\left\|\frac{\partial w_{1}}{\partial x_{1}}\right\|_{0,3 / 2} \leq\left\|\frac{\partial w}{\partial x_{1}}\right\|_{0, q}^{3 / q^{\prime}}\|\nabla w\|_{0,3 q /(3-q)}^{1-3 / q^{\prime}} \leq \lambda^{-2 / q^{\prime}-1 / 3}\|w\|_{\mathbf{z}_{2}, \lambda}, \\
\left\|\nabla w_{2}\right\|_{0,3 / 2} \leq\left\|\nabla w_{2}\right\|_{0, q}^{3 / q^{\prime}}\left\|\nabla w_{2}\right\|_{0,3 q /(3-q)}^{1-3 / q^{\prime}} \leq \lambda^{-2 / q^{\prime}-1 / 3}\|w\|_{\mathbf{z}_{2}, \lambda}, \\
\left\|v_{2}\right\|_{0,3} \leq\left\|v_{2}\right\|_{0,2 q /(2-q)}^{6 / q^{\prime}}\|v\|_{0,3 q /(3-2 q)}^{1-6 / q^{\prime}} \leq \lambda^{-2 / q^{\prime}-2 / 3}\|w\|_{\mathbf{z}_{2}, \lambda}
\end{gathered}
$$

with $1 / q^{\prime}=1-1 / q$. The estimates of $\|(1+\sigma) v \cdot \nabla w\|_{0, q}$ and $\|(1+\sigma) v \nabla \cdot w\|_{0, q}$ are thus an easy consequence of (8.3), (8.4) and the above three inequalities. For $\|\nabla \sigma w \otimes v\|_{0, q}$, we have

$$
\begin{gathered}
\|\nabla \sigma w \otimes v\|_{0, q} \leq\|\nabla \sigma\|_{0, q}|v|_{\mathcal{C}^{0}}|w|_{\mathcal{C}^{0}} \\
\leq c \lambda^{-1}\|w\|_{\mathbf{z}_{2}, \lambda}\|v\|_{\mathbf{z}_{2}, \lambda}\|\sigma\|_{\mathbf{z}_{1}, \lambda}
\end{gathered}
$$

Estimates of $\|\nabla((1+\sigma) v \cdot \nabla w)\|_{0, q},\|\nabla((1+\sigma) v \nabla \cdot w)\|_{0, q},\|\nabla(\nabla \sigma v \otimes w)\|_{0, q}$ are even easier and therefore left to the reader.

Estimate $(8.1)_{4}$ is obvious since

$$
\lambda\left\|\frac{\partial(\sigma v)}{\partial x_{1}}\right\|_{0, q} \leq \lambda\left(|v|_{\mathcal{C}^{0}}\|\nabla \sigma\|_{0, q}+|\sigma|_{\mathcal{C}^{0}}\left\|\frac{\partial v}{\partial x_{1}}\right\|_{0, q}\right) \leq c \lambda^{1 / 3}\|\sigma\|_{\mathbf{z}_{1}, \lambda}\|v\|_{\mathbf{z}_{2, \lambda}}
$$

an analogous estimate for $\lambda\left\|\frac{\partial \nabla(\sigma v)}{\partial x_{1}}\right\|_{0, q}$ holds.

Last, we prove the estimates $(8.1)_{5-6}$ :

$$
\begin{gathered}
\|(1+\sigma) f\|_{0, q} \leq\left(1+|\sigma|_{\mathcal{C}^{0}}\right)\|f\|_{0, q} \leq c\left(\|f\|_{0, q}+\lambda^{-1 / 3}\|\sigma\|_{\mathbf{z}_{1, \lambda}}\|f\|_{0, q}\right), \\
\|\nabla((1+\sigma) f)\|_{0, q} \leq\left(1+|\sigma|_{\mathcal{C}^{0}}\right)\|\nabla f\|_{0, q}+|\nabla \sigma|_{\mathcal{C}^{0}}\|f\|_{0, q} \\
\leq c\left(\|\nabla f\|_{0, q}+\lambda^{-1 / 3}\|\sigma\|_{\mathbf{z}_{1, \lambda}}\|f\|_{1, q}\right) .
\end{gathered}
$$


Proof of Lemma 8.1 is so completed.

Lemma 8.2. Let $\lambda \in(0,1), 1<q<6 / 5, k=1, \ldots$, and $p \geq 3 q /(3-2 q)$. Assume that $w \in \mathbf{Z}_{2}, v \in \mathbf{Z}_{2}, \sigma \in \mathbf{Z}_{1}, f \in W^{k, p}(\Omega)$. Then

$$
\begin{gathered}
\|(1+\sigma) w \nabla v\|_{k, p} \leq c \lambda^{-1-2(1-1 / q)}\left(1+\lambda^{-1 / 3}\|\sigma\|_{\mathbf{z}_{1}, \lambda}\right)\|w\|_{\mathbf{z}_{2, \lambda},}\|v\|_{\mathbf{z}_{2}, \lambda}, \\
\|\nabla \sigma w \otimes v\|_{k, p} \leq c \lambda^{-1-2(1-1 / q)}\|\sigma\|_{\mathbf{z}_{1}, \lambda}\|w\|_{\mathbf{z}_{2}, \lambda}\|v\|_{\mathbf{z}_{2}, \lambda}, \\
\|\sigma v\|_{k+1, p} \leq c \lambda^{-1-2(1-1 / q)}\|\sigma\|_{\mathbf{z}_{1}, \lambda}\|v\|_{\mathbf{Z}_{2}, \lambda} \\
\|(1+\sigma) f\|_{k, p} \leq c\left(1+\lambda^{-1 / 3}\|\sigma\|_{\mathbf{z}_{1}, \lambda}\right)\|f\|_{k, p} .
\end{gathered}
$$

Proof. Proof of the estimates $(8.8)_{1-3}$ :

$$
\begin{gathered}
\|(1+\sigma) w \nabla v\|_{k, p} \leq\left(1+|\sigma|_{\mathcal{C}^{k}}\right)|w|_{\mathcal{C}^{k}}\|\nabla v\|_{k, p} \\
\leq c \lambda^{-2 / 3}\left(1+\lambda^{-1 / 3}\|\sigma\|_{\mathbf{z}_{1, \lambda}, \lambda}\right)\|w\|_{\mathbf{z}_{2, \lambda},}\|v\|_{\mathbf{z}_{2}, \lambda} \\
\|\nabla \sigma w v\|_{k, p} \leq c|w|_{\mathcal{C}^{k}}|v|_{\mathcal{C}^{k}}\|\nabla \sigma\|_{k, p} \\
\leq c \lambda^{-1}\|w\|_{\mathbf{z}_{2, \lambda}}\|v\|_{\mathbf{z}_{2, \lambda}}\|\sigma\|_{\mathbf{z}_{1}, \lambda} \\
\|\sigma v\|_{k+1, p} \leq c \lambda^{-2 / 3}\|\sigma\|_{\mathbf{z}_{1, \lambda}}\|v\|_{\mathbf{z}_{2}, \lambda} .
\end{gathered}
$$

Proof of the estimates $(8.8)_{4}$ :

$$
\|(1+\sigma) f\|_{k, p} \leq\left(1+|\sigma|_{\mathcal{C}^{k}}\right)\|f\|_{k, p} \leq c\left(1+\lambda^{-1 / 3}\|\sigma\|_{\mathbf{z}_{1, \lambda}}\right)\|f\|_{k, p} .
$$

Lemma 8.2 is thus verified.

Applying Theorem 5.2 to the system $\left(2.4^{\prime}\right)$ with $\mathcal{F}=F(\tau, w)\left(\right.$ see $\left(2.3_{2}\right)$ ) and using Lemmas 8.1, 8.2 for the estimating of the corresponding norms of $F$, one gets

$$
\begin{gathered}
\|(\sigma, v)\|_{\mathbf{z}, \lambda} \leq c_{3}\left\{\left(1+\lambda^{-1 / 3}\|\tau\|_{\mathbf{z}_{1}, \lambda}\right)\|f\|_{\mathbf{L}}\right. \\
+\lambda^{-1-2(1-1 / q)}\left(1+\lambda^{-1 / 3}\|\tau\|_{\mathbf{z}_{1}, \lambda}\right)\|(\tau, w)\|_{\mathbf{Z}, \lambda}^{2} \\
\left.+|\ln \lambda|^{-1} \lambda^{1+2(1-1 / q)}\right\} .
\end{gathered}
$$

(Recall that $c_{3}$ is independent of $\lambda$.) Let $\lambda<\gamma_{0}<1, \gamma_{1}=\beta \lambda^{\kappa^{\prime}}, \gamma_{2}=\alpha \lambda^{\kappa}$ with $\kappa, \kappa^{\prime}>0$ and $\lambda, \alpha, \beta$ sufficiently small such that $\alpha \lambda^{-2 / 3+\kappa}+\lambda^{2 / 3}<\gamma$ (where $\gamma$ is defined in Theorem 5.2). Let

$$
(\tau, w) \in B_{\gamma_{2}}(0)=\left\{(\tau, w) \in Z, \quad\|(\tau, w)\|_{\mathbf{z}, \lambda} \leq \gamma_{2}\right\} .
$$


Then there exist $\alpha, \beta, \gamma_{0}, \kappa, \kappa^{\prime}$ such that for $\lambda<\gamma_{0}$, it is also $(\sigma, v) \in B_{\gamma_{2}}(0)$. Namely, we require that

$$
\begin{gathered}
c_{3}\left(\beta\left(1+\alpha \lambda^{\kappa-1 / 3}\right) \lambda^{\kappa^{\prime}}+\alpha^{2}\left(1+\alpha \lambda^{\kappa-1 / 3}\right) \lambda^{2 \kappa-1-2(1-1 / q)}\right. \\
\left.+|\ln \lambda|^{-1} \lambda^{1+2(1-1 / q)}\right) \leq \alpha \lambda^{\kappa} .
\end{gathered}
$$

An easy calculation shows that it is possible for $\kappa=1+2(1-1 / q), \kappa^{\prime} \geq \kappa$ and $\alpha, \beta, \gamma_{0}$ sufficiently small. ${ }^{5}$ For such $\alpha, \beta, \gamma_{0}, \kappa, \kappa^{\prime}$ and corresponding $\gamma_{0}, \gamma_{1}, \gamma_{2}$, we have

$$
\|(\sigma, v)\|_{\mathbf{z}, \lambda} \leq \gamma_{2} .
$$

Let $(\sigma, v),\left(\sigma^{(1)}, v^{(1)}\right)$ be two solutions of the problem $\left(2.4^{\prime}\right)$ corresponding to the given quantities $(\tau, w), \mathcal{F}=F(\tau, w)$, and $\left(\tau^{(1)}, w^{(1)}\right), \mathcal{F}=F\left(\tau^{(1)}, w^{(1)}\right)$, respectively. Set $(\tilde{\tau}, \tilde{w})=(\tau, w)-\left(\tau^{(1)}, w^{(1)}\right),(\tilde{\sigma}, \tilde{v})=(\sigma, v)-\left(\sigma^{(1)}, v^{(1)}\right)$, $F=F(\tau, w), F^{(1)}=F\left(\tau^{(1)}, w^{(1)}\right), \tilde{F}=F-F^{(1)}$. Then $(\tilde{\sigma}, \tilde{v})$ satisfies in virtue of (2.3), the following system

$$
\begin{gathered}
-\mu_{1} \Delta \tilde{v}-\left(\mu_{1}+\mu_{2}\right) \nabla \operatorname{div} \tilde{v}+v_{\infty} \frac{\partial \tilde{v}}{\partial x_{1}}+\nabla \tilde{\sigma}=\tilde{F}, \\
\operatorname{div} \tilde{v}+\operatorname{div}\left(\left(w^{(1)}+\mathbf{v}_{\infty}\right) \tilde{\sigma}\right)=-\operatorname{div}(\tilde{w} \sigma)=\tilde{h}, \\
\left.\tilde{v}\right|_{\partial \Omega}=0, \quad \tilde{v} \rightarrow 0, \tilde{\sigma} \rightarrow 0 \text { as }|x| \rightarrow \infty .
\end{gathered}
$$

It is easy to verify (for $(\tau, w),\left(\tau^{(1)}, w^{(1)}\right) \in B_{\gamma_{2}}(0)$ )

$$
\begin{gathered}
\|\tilde{F}\|_{\mathbf{L}^{\prime}} \leq c \lambda^{-1-2(1-1 / q)}\left(1+\lambda^{-1 / 3} \gamma_{2}\right)\left(\gamma_{2}+\|f\|_{\mathbf{L}}\right)\|(\tilde{\tau}, \tilde{w})\|_{\mathbf{Z}^{\prime}, \lambda}, \\
\|\tilde{h}\|_{\mathbf{D}^{\prime}} \leq c \lambda^{-2 / 3} \gamma_{2}\|\tilde{w}\|_{\mathbf{Z}_{2}^{\prime}, \lambda} .
\end{gathered}
$$

Indeed, the estimates for $\|\tilde{F}\|_{0, q},\|\tilde{F}\|_{k-1, p}, k \geq 1$ follow similarly as Lemmas 8.1, 8.2. Estimates for $\|\tilde{h}\|_{0, q},|\tilde{h}|_{*, p},|\tilde{h}|_{*, 3 q /(3-2 q)}$ are consequence of the inequalities below:

$$
\begin{gathered}
\|\operatorname{div}(\tilde{w} \sigma)\|_{0, q} \leq\|\sigma \nabla \cdot \tilde{w}\|_{0, q}+\|\tilde{w} \cdot \nabla \sigma\|_{0, q} \\
\leq\|\nabla \tilde{w}\|_{0,3 q /(3-q)}\|\sigma\|_{0,3}+|\tilde{w}|_{C^{0}}\|\nabla \sigma\|_{0, q} ; \\
|\operatorname{div}(\tilde{w} \sigma)|_{*, p} \leq\|\tilde{w} \sigma\|_{0, p} \leq|\tilde{w}|_{C^{0}}\|\sigma\|_{0, p} ; \\
|\operatorname{div}(\tilde{w} \sigma)|_{*, 3 q /(3-2 q)} \leq\|\tilde{w} \sigma\|_{0,3 q /(3-2 q)} \leq|\tilde{w}|_{C^{0}}\left(\|\sigma\|_{0,2 q /(2-q)}+\|\sigma\|_{0, p}\right) .
\end{gathered}
$$

${ }^{5}$ Certainly $B_{\gamma_{2}}(0)$ is not empty. Indeed, if $(\sigma, v)$ is a solution of problem (2.4) with $\mathcal{F}=0, g=0, w$ satisfying the condition (5.6) and $\Psi=\mathbf{v}_{\infty}$, then $(\sigma, v)$ is in $B_{\gamma_{2}}(0)$ provided $\gamma_{2} \geq c_{2}^{\prime}|\ln \lambda|^{-1} \lambda^{1+2(1-1 / q)}$, see Theorem 5.2. 
Estimates for $\|\nabla \tilde{h}\|_{0, q},\|\tilde{h}\|_{k, p}$ are even easier. Estimate (8.13) is thus verified. Applying Theorem 5.2 (estimate with $\mathbf{Z}^{\prime}, \mathbf{L}^{\prime}, \mathbf{B}^{\prime}, \mathbf{D}^{\prime}$ ) to (8.12), we find

$$
\begin{aligned}
&\|(\tilde{\sigma}, \tilde{v})\|_{\mathbf{Z}^{\prime}, \lambda} \leq c \lambda^{-1-2(1-1 / q)}\left(1+\lambda^{-1 / 3} \gamma_{2}\right)\left(\gamma_{2}+\gamma_{1}\right) \\
& \times\left(\|(\tilde{\tau}, \tilde{w})\|_{\mathbf{Z}^{\prime}, \lambda}+\|(\tilde{\sigma}, \tilde{v})\|_{\mathbf{Z}^{\prime}, \lambda}\right) .
\end{aligned}
$$

This last inequality implies, by the same reasoning as in (8.10), the existence of a $\delta \in(0,1)$, such that (recall we choose $\gamma_{1}=\beta \lambda^{\kappa^{\prime}}, \gamma_{2}=\alpha \lambda^{\kappa}$ with $\alpha, \beta$, and $\lambda$ sufficiently small and $\left.\kappa=1+2(1-1 / q), \kappa^{\prime} \geq \kappa\right)$

$$
\|(\tilde{\sigma}, \tilde{v})\|_{\mathbf{Z}^{\prime}, \lambda} \leq \delta\|(\tilde{\tau}, \tilde{w})\|_{\mathbf{Z}^{\prime}, \lambda} .
$$

Now, by a standard version of the Banach contraction principle for closed subsets of Banach spaces (see Zeidler [58]), we get the existence of a fixed point $(\sigma, v) \in B_{\gamma_{2}}(0) \subset Z$, which obviously satisfies system of equations (2.3). The estimate (5.4) follows from (8.9) written in the fixed point. Theorem 5.1 is thus proved.

\section{Appendix.}

First, consider in the whole space $\mathbb{R}^{2}$ the problem

$$
-\Delta u+\nabla \Pi+\lambda \frac{\partial u}{\partial x_{1}}=\varpi
$$

$$
\operatorname{div} u=h \text {. }
$$

Put

$$
u(x)=\frac{1}{2 \pi} \int_{\mathbb{R}^{2}} e^{i x \cdot \xi} \mathcal{V}(\xi) d \xi, \quad \Pi(x)=\frac{1}{2 \pi} \int_{\mathbb{R}^{2}} e^{i x \cdot \xi} \mathcal{P}(\xi) d \xi
$$

where ${ }^{6}$

$$
\begin{gathered}
\mathcal{V}_{m}(\xi)=-\frac{\xi_{m} \xi_{k}-|\xi|^{2} \delta_{m k}}{|\xi|^{2}\left(|\xi|^{2}-i \xi_{1} \lambda\right)} \hat{\varpi}_{k}(\xi)+i \frac{\xi_{m}}{|\xi|^{2}} \hat{h}(\xi), \\
\mathcal{P}(\xi)=i \hat{\varpi}_{m}(\xi) \frac{\xi_{m}}{|\xi|^{2}}+\left(-\frac{i \xi_{1} \lambda}{|\xi|^{2}}+1\right) \hat{h}(\xi)
\end{gathered}
$$

(here $\hat{a}$ denotes the Fourier transform of $\left.a, \hat{a}(\xi)=\frac{1}{2 \pi} \int_{\mathbb{R}^{2}} e^{-i x \cdot \xi} a(x) d x\right)$. If $\varpi, h \in \mathcal{C}_{0}^{\infty}\left(\mathbb{R}^{2}\right)$ then $\hat{\varpi}, \hat{h} \in \mathcal{S}\left(\mathbb{R}^{2}\right)$ (the space of rapidly decreasing functions); it is not hard to show that, in this case, (A.2) defines a $\mathcal{C}^{\infty}$-solution of the

\footnotetext{
${ }^{6}$ We use the Einstein summation convention over repeated indexes.
} 
problem (A.1), see Galdi [24], Vol. I, $\S 7.2$ or [23]. Due to the Lizorkin Theorem (see again Galdi [24], Vol. I, §7.2), we have the estimate

$$
\left\|\nabla^{2} u\right\|_{l, t, \mathbb{R}^{2}}+\|\nabla \Pi\|_{l, t, \mathbb{R}^{2}} \leq c\left[\|\varpi\|_{l, t, \mathbb{R}^{2}}+\|h\|_{l+1, t, \mathbb{R}^{2}}\right]
$$

which is valid for $l=0, \ldots, k$ and $1<t<\infty$. Moreover, if $1<t<3 / 2$, one discovers again by the Lizorkin theorem

$$
\begin{gathered}
\lambda^{2 / 3}\|u\|_{0,3 t /(3-2 t), \mathbb{R}^{2}}+\lambda\left(\left\|\frac{\partial u}{\partial x_{1}}\right\|_{0, t, \mathbb{R}^{2}}+\left\|u_{2}\right\|_{0,2 t /(2-t), \mathbb{R}^{2}}\right. \\
\left.+\left\|\nabla u_{2}\right\|_{0, t, \mathbb{R}^{2}}\right)+\lambda^{1 / 3}\left[\|\nabla u\|_{0,3 t /(3-t), \mathbb{R}^{2}}+\|\Pi\|_{0,2 t /(2-t), \mathbb{R}^{2}}\right] \\
\leq c\left[\|\varpi\|_{0, t, \mathbb{R}^{2}}+\|h\|_{1, t, \mathbb{R}^{2}}\right] .
\end{gathered}
$$

Therefore, we have the following two lemmas, cf. Galdi [24], Vol I, §7.2:

Lemma A.1. Let $1<q, p<+\infty, k=0,1, \ldots$ and

$$
\varpi \in W^{1, q}\left(\mathbb{R}^{2}\right) \cap W^{k, p}\left(\mathbb{R}^{2}\right), h \in W^{2, q}\left(\mathbb{R}^{2}\right) \cap W^{k+1, p}\left(\mathbb{R}^{2}\right) .
$$

Then there exists a solution

$$
\begin{aligned}
& u \in W_{\mathrm{loc}}^{3, q}\left(\mathbb{R}^{2}\right) \cap W_{\mathrm{loc}}^{k+2, p}\left(\mathbb{R}^{2}\right), \nabla^{2} u \in W^{1, q}\left(\mathbb{R}^{2}\right) \cap W^{k, p}\left(\mathbb{R}^{2}\right), \\
& \Pi \in W_{\mathrm{loc}}^{2, q}\left(\mathbb{R}^{2}\right) \cap W_{\mathrm{loc}}^{k+1, p}\left(\mathbb{R}^{2}\right), \quad \nabla \Pi \in W^{1, q}\left(\mathbb{R}^{2}\right) \cap W^{k, p}\left(\mathbb{R}^{2}\right),
\end{aligned}
$$

to the problem (A.1) satisfying the representation formulas (A.2), (A.3). If $(\tilde{u}, \tilde{\Pi})$ is another solution in the same class, then $\tilde{u}=u+a_{0}+a_{j} x_{j}, \tilde{\Pi}=\Pi+b$, $a_{\alpha} \in \mathbb{R}^{2}, b \in \mathbb{R}^{1}$. Moreover we have the estimate

$$
\begin{gathered}
\left\|\nabla^{2} u\right\|_{1, q, \mathbb{R}^{2}}+\|\nabla \Pi\|_{1, q, \mathbb{R}^{2}}+\left\|\nabla^{2} u\right\|_{k, p, \mathbb{R}^{2}}+\|\nabla \Pi\|_{k, p, \mathbb{R}^{2}} \\
\leq c\left[\|\varpi\|_{1, q, \mathbb{R}^{2}}+\|h\|_{2, q, \mathbb{R}^{2}}+\|\varpi\|_{k, p, \mathbb{R}^{2}}+\|h\|_{k+1, p, \mathbb{R}^{2}}\right] .
\end{gathered}
$$

Lemma A.2. If $1<q<3 / 2$, and $\varpi \in L^{q}\left(\mathbb{R}^{2}\right), h \in W^{1, q}\left(\mathbb{R}^{2}\right)$, then there exists a unique solution

$$
\begin{gathered}
u \in L^{3 q /(3-2 q)}\left(\mathbb{R}^{2}\right), \nabla u \in L^{3 q /(3-q)}\left(\mathbb{R}^{2}\right), \nabla u_{2}, \frac{\partial u}{\partial x_{1}} \in L^{q}\left(\mathbb{R}^{2}\right), \\
\nabla^{2} u \in L^{q}\left(\mathbb{R}^{2}\right), u_{2} \in L^{2 q /(2-q)}\left(\mathbb{R}^{2}\right), \Pi \in L^{2 q /(2-q)}\left(\mathbb{R}^{2}\right), \nabla \Pi \in L^{q}\left(\mathbb{R}^{2}\right)
\end{gathered}
$$


to the problem (A.1) satisfying the estimate

$$
\begin{gathered}
\lambda^{2 / 3}\|u\|_{0,3 q /(3-2 q), \mathbb{R}^{2}}+\lambda\left(\left\|\frac{\partial u}{\partial x_{1}}\right\|_{0, q, \mathbb{R}^{2}}+\left\|u_{2}\right\|_{0,2 q /(2-q), \mathbb{R}^{2}}\right. \\
\left.+\left\|\nabla u_{2}\right\|_{0, q, \mathbb{R}^{2}}\right)+\lambda^{1 / 3}\left[\|\nabla u\|_{0,3 q /(3-q), \mathbb{R}^{2}}+\left\|\nabla^{2} u\right\|_{0, q, \mathbb{R}^{2}}+\|\nabla \Pi\|_{0, q, \mathbb{R}^{2}}\right. \\
\left.+\|\Pi\|_{0,2 q /(2-q), \mathbb{R}^{2}}\right] \leq c\left[\|\varpi\|_{0, q, \mathbb{R}^{2}}+\|h\|_{1, q, \mathbb{R}^{2}}\right] .
\end{gathered}
$$

In Lemmas A.1, A.2 and in the estimates (A.4), (A.5), $c$ is a positive constant which depends, in particular, of $\lambda_{0}\left(\lambda \in\left(0, \lambda_{0}\right)\right)$. We point out that it is independent of $\lambda$. With these results at hand, we can proceed to the proof of Lemma 6.4.

Proof of Lemma 6.4.

We put in (6.8), without loss of generality, $\mu=1$ and we suppose that $\lambda<1$, for example. We introduce a parameter $\chi$ which is equal to 0 provided $\varpi \in W^{1, q}(\Omega), h \in W^{2, q}(\Omega), \psi \in W^{3-1 / q, q}(\partial \Omega)$ and $\chi=1$ if in the hypothesis the $W^{k, p}$-norms are present. Moreover, for two sets $A, B$, we denote $A \cap$ $\chi B=A$ if $\chi=0$ and $A \cap \chi B=A \cap B$ if $\chi=1$. Take a (unique) solution $(u, \Pi)$ of the problem (6.8) belonging to class (6.9), see Lemma 6.3. Since

$$
\begin{gathered}
\varpi \in W^{1, q}(\Omega) \cap \chi W^{k, p}(\Omega), h \in W^{2, q}(\Omega) \cap \chi W^{k+1, p}(\Omega), \\
\psi \in W^{3-1 / q, q}(\partial \Omega) \cap \chi W^{k+2-1 / p, p}(\partial \Omega),
\end{gathered}
$$

we also have

$$
u \in W_{\mathrm{loc}}^{3, q}(\bar{\Omega}) \cap \chi W_{\mathrm{loc}}^{k+2, p}(\bar{\Omega}), \Pi \in W_{\mathrm{loc}}^{2, q}(\bar{\Omega}) \cap \chi W_{\mathrm{loc}}^{k+1, p}(\bar{\Omega}),
$$

cf. Galdi [24], Vol. I, §4.2. Define

$$
u^{R}=u\left(1-\Phi_{R}\right), \Pi^{R}=\Pi\left(1-\Phi_{R}\right), u_{R}=u \Phi_{R}, \Pi_{R}=\Pi \Phi_{R},
$$

where $\Phi_{R}$ is given by:

$$
\Phi_{R}(x)=\Phi\left(\frac{x}{R}\right)
$$

where

$$
\begin{gathered}
\Phi \in C_{0}^{\infty}\left(\mathbb{R}^{2}\right), \\
\Phi(x)=\left\{\begin{array}{lll}
1, & \text { if } & x \in B_{1}(0), \\
0, & \text { if } & x \in B^{2}(0) .
\end{array}\right.
\end{gathered}
$$


Clearly, we have

$$
\left|\nabla^{\alpha} \Phi_{R}\right| \leq c R^{-\alpha}, \operatorname{supp}\left(\nabla^{\alpha} \Phi_{R}\right)=B_{2 R}(0)-B_{R}(0) .
$$

The couple $\left(\Pi^{R}, u^{R}\right)$ satisfies the equations

$$
\begin{gathered}
-\Delta u^{R}+\lambda \frac{\partial u^{R}}{\partial x_{1}}+\nabla \Pi^{R}=\varpi\left(1-\Phi_{R}\right)+\varpi_{c}, \\
\operatorname{div} u^{R}=h\left(1-\Phi_{R}\right)+h_{c},
\end{gathered}
$$

in $\mathbb{R}^{2}$. Here

(A.8) $\varpi_{c}=2 \nabla \Phi_{R} \cdot \nabla u+u \Delta \Phi_{R}-\lambda \frac{\partial \Phi_{R}}{\partial x_{1}} u-\nabla \Phi_{R} \Pi, \quad h_{c}=-\nabla \Phi_{R} \cdot u$.

We have by the direct calculation

$$
\begin{gathered}
\left\|\varpi\left(1-\Phi_{R}\right)\right\|_{j, s, \mathbb{R}^{2}} \leq c\|\varpi\|_{j, s, \Omega}, \quad\left\|h\left(1-\Phi_{R}\right)\right\|_{j+1, s, \mathbb{R}^{2}} \leq c\|h\|_{j+1, s, \Omega} \\
\left\|\varpi_{c}\right\|_{j, s, \mathbb{R}^{2}} \leq c\left(\|u\|_{j+1, s, \Omega_{2 R}}+\|\Pi\|_{j, s, \Omega_{2 R}}\right), \quad\left\|h_{c}\right\|_{j+1, s, \mathbb{R}^{2}} \leq c\|u\|_{j+1, s, \Omega_{2 R}}
\end{gathered}
$$

where $s=q$ and $j=1$ or $s=p$ and $j=k$. (Naturally $c$ depends of $k, q, p$ and R.) Thus, in virtue of Lemma A.1

$$
\begin{gathered}
\lambda^{1 / 3}\left(\left\|\nabla^{2} u^{R}\right\|_{1, q, \mathbb{R}^{2}}+\chi\left\|\nabla^{2} u^{R}\right\|_{k, p, \mathbb{R}^{2}}\right. \\
\left.+\left\|\nabla \Pi^{R}\right\|_{1, q, \mathbb{R}^{2}}+\left\|\Pi^{R}\right\|_{0,2 q /(2-q), \mathbb{R}^{2}}+\chi\left\|\nabla \Pi^{R}\right\|_{k, p, \mathbb{R}^{2}}\right) \\
\leq c\left[\|\varpi\|_{1, q, \mathbb{R}^{2}}+\chi\|\varpi\|_{k, p, \mathbb{R}^{2}}+\|h\|_{2, q, \mathbb{R}^{2}}+\chi\|h\|_{k+1, p, \mathbb{R}^{2}}\right. \\
\left.+\lambda^{1 / 3}\left(\|u\|_{2, q, \Omega_{2 R}}+\chi\|u\|_{k+1, p, \Omega_{2 R}}+\|\Pi\|_{1, q, \Omega_{2 R}}+\chi\|\Pi\|_{k, p, \Omega_{2 R}}\right)\right] .
\end{gathered}
$$

By the Sobolev imbeddings and the interpolation (recall that $\frac{2 q}{2-q} \leq p$ ), one gets directly

$$
\left\|\nabla u^{R}\right\|_{0, p, \mathbb{R}^{2}}+\left\|\Pi^{R}\right\|_{0, p, \mathbb{R}^{2}} \leq c\left(\left\|\nabla^{2} u^{R}\right\|_{1, q, \mathbb{R}^{2}}+\left\|\nabla \Pi^{R}\right\|_{1, q, \mathbb{R}^{2}}\right) .
$$

This furnishes, in turn with the previous estimate

$$
\begin{gathered}
\lambda^{1 / 3}\left(\left\|\nabla^{2} u^{R}\right\|_{1, q, \mathbb{R}^{2}}+\chi\left\|\nabla u^{R}\right\|_{k+1, p, \mathbb{R}^{2}}\right. \\
\left.+\left\|\nabla \Pi^{R}\right\|_{1, q, \mathbb{R}^{2}}+\left\|\Pi^{R}\right\|_{0,2 q /(2-q), \mathbb{R}^{2}}+\chi\left\|\Pi^{R}\right\|_{k, p, \mathbb{R}^{2}}\right) \\
\leq c\left[\|\varpi\|_{1, q, \mathbb{R}^{2}}+\chi\|\varpi\|_{k, p, \mathbb{R}^{2}}+\|h\|_{2, q, \mathbb{R}^{2}}+\chi\|h\|_{k+1, p, \mathbb{R}^{2}}\right. \\
\left.+\lambda^{1 / 3}\left(\|u\|_{2, q, \Omega_{2 R}}+\chi\|u\|_{k+1, p, \Omega_{2 R}}+\|\Pi\|_{1, q, \Omega_{2 R}}+\chi\|\Pi\|_{k, p, \Omega_{2 R}}\right)\right] .
\end{gathered}
$$


On the other hand

$$
\begin{gathered}
-\Delta u_{R}+\nabla \Pi_{R}=\varpi \Phi_{R}+\varpi_{c}^{\prime} \quad \text { in } \Omega_{2 R}, \\
\operatorname{div} u_{R}=h \Phi_{R}-h_{c} \quad \text { in } \quad \Omega_{2 R}, \\
\left.u_{R}\right|_{\partial \Omega}=\psi,\left.\quad u_{R}\right|_{\partial B_{2 R}}=0,
\end{gathered}
$$

where $h_{c}$ is given by (A.8) and

$$
\varpi_{c}^{\prime}=-2 \nabla \Phi_{R} \cdot \nabla u-u \Delta \Phi_{R}+\lambda \frac{\partial u}{\partial x_{1}} \Phi_{R}+\nabla \Phi_{R} \Pi .
$$

By easy calculation, one obtains

$$
\begin{gathered}
\left\|\varpi \Phi_{R}\right\|_{j, s, \Omega_{2 R}} \leq c\|\varpi\|_{j, s, \Omega}, \quad\left\|h \Phi_{R}\right\|_{j+1, s, \Omega_{2 R}} \leq c\|h\|_{j+1, s, \Omega}, \\
\left\|\varpi_{c}^{\prime}\right\|_{j, s, \Omega_{2 R}} \leq c\left(\|u\|_{j+1, s, \Omega_{2 R}}+\|\Pi\|_{j, s, \Omega_{2 R}}\right) \\
\left\|h_{c}\right\|_{j+1, s, \Omega_{2 R}} \leq c\|u\|_{j+1, s, \Omega_{2 R}}
\end{gathered}
$$

where $s=q$ and $j=1$ or $s=p$ and $j=\mathrm{k}$. Thus, by the classical theorem of Cattabriga [11] for the Stokes problem in bounded domains, we get the following estimate

$$
\begin{gathered}
\lambda^{1 / 3}\left(\left\|u_{R}\right\|_{3, q, \Omega_{2 R}}+\chi\left\|u_{R}\right\|_{k+2, p, \Omega_{2 R}}\right. \\
\left.+\left\|\nabla \Pi_{R}\right\|_{1, q, \Omega_{2 R}}+\chi\left\|\nabla \Pi_{R}\right\|_{k, p, \Omega_{2 R}}\right) \\
\leq c \lambda^{1 / 3}\left[\|\varpi\|_{1, q, \Omega}+\chi\|\varpi\|_{k, p, \Omega}+\|h\|_{2, q, \Omega}+\chi\|h\|_{k+1, p, \Omega}\right. \\
+\|u\|_{2, q, \Omega_{2 R}}+\chi\|u\|_{k+1, p, \Omega_{2 R}}+\|\Pi\|_{1, q, \Omega_{2 R}}+\chi\|\Pi\|_{k, p, \Omega_{2 R}} \\
\left.+\chi\|\psi\|_{k+2-1 / p, p, \partial \Omega}+\|\psi\|_{3-1 / q, q, \partial \Omega}\right] .
\end{gathered}
$$

Due to the Poincaré inequality (recall that $\left.\Pi\right|_{\partial B_{2 R}}=0$ ), $\left\|\Pi_{R}\right\|_{k+1, p, \Omega_{2 R}} \leq c$ $\left\|\nabla \Pi_{R}\right\|_{k, p, \Omega_{2 R}}$. Adding (A.9), (A.10) and (6.10), we thus get, in particular

$$
\begin{gathered}
\lambda^{1 / 3}\left[\|\nabla u\|_{0,3 q /(3-q), \Omega}+\left\|\nabla^{2} u\right\|_{1, q, \Omega}+\chi\|\nabla u\|_{k+1, p, \Omega}\right. \\
\left.+\|\nabla \Pi\|_{1, q, \Omega}+\|\Pi\|_{0,2 q /(2-q), \Omega}+\chi\|\Pi\|_{k+1, p, \Omega}\right] \\
\leq c\left[\|\varpi\|_{1, q, \Omega}+\chi\|\varpi\|_{k, p, \Omega}+\|h\|_{2, q, \Omega}+\chi\|h\|_{k+1, p, \Omega}\right. \\
+\lambda^{1 / 3}\left(\|u\|_{2, q, \Omega_{2 R}}+\chi\|u\|_{k+1, p, \Omega_{2 R}}+\|\Pi\|_{1, q, \Omega_{2 R}}+\chi\|\Pi\|_{k, p, \Omega_{2 R}}\right) \\
\left.+\lambda^{1 / 3}\left(\chi\|\psi\|_{k+2-1 / p, p, \partial \Omega}+\|\psi\|_{3-1 / q, q, \partial \Omega}\right)\right] .
\end{gathered}
$$


By the Sobolev imbeddings, it holds in particular

$$
\begin{gathered}
\|u\|_{2, q, \Omega_{2 R}} \leq c\left[\|\nabla u\|_{0,3 q /(3-q), \Omega}+\left\|\nabla^{2} u\right\|_{0, q, \Omega}\right] \\
\|\Pi\|_{1, q, \Omega_{2 R}} \leq c\left[\|\nabla \Pi\|_{0, q, \Omega}+\|\Pi\|_{0,2 q /(2-q), \Omega}\right]
\end{gathered}
$$

These estimates, (A.11) and Lemma 6.3, furnish

$$
\begin{gathered}
\lambda^{1 / 3}\left[\|\nabla u\|_{0,3 q /(3-q), \Omega}+\left\|\nabla^{2} u\right\|_{1, q, \Omega}+\|\nabla \Pi\|_{1, q, \Omega}+\|\Pi\|_{0,2 q /(2-q), \Omega}\right] \\
\leq c\left[\|\varpi\|_{1, q, \Omega}+\|h\|_{2, q, \Omega}+\lambda^{1 / 3}\|\psi\|_{3-1 / q, q, \partial \Omega}\right] .
\end{gathered}
$$

Further, we have, again by the Sobolev imbedding (recall that $p \in\left[\frac{2 q}{2-q},+\infty\right)$ ):

$$
\begin{gathered}
\|u\|_{1, p, \Omega_{2 R}} \leq c\left[\|\nabla u\|_{0,3 q /(3-q), \Omega}+\left\|\nabla^{2} u\right\|_{1, q, \Omega}\right] \\
\|\Pi\|_{0, p, \Omega_{2 R}} \leq c\left[\|\nabla \Pi\|_{1, q, \Omega}+\|\Pi\|_{0,2 q /(2-q), \Omega}\right] .
\end{gathered}
$$

By these facts and (A.11)

$$
\begin{gathered}
\lambda^{1 / 3}\left[\|\nabla u\|_{0,3 q /(3-q), \Omega}+\left\|\nabla^{2} u\right\|_{1, q, \Omega}+\|\nabla u\|_{1, p, \Omega}\right. \\
\left.+\|\nabla \Pi\|_{1, q, \Omega}+\|\Pi\|_{0,2 q /(2-q), \Omega}+\|\Pi\|_{1, p, \Omega}\right] \\
\leq c\left[\|\varpi\|_{1, q, \Omega}+\|\varpi\|_{0, p, \Omega}+\|h\|_{2, q, \Omega}+\|h\|_{1, p, \Omega}\right. \\
\left.\quad+\lambda^{1 / 3}\left(\|\psi\|_{2-1 / p, p, \partial \Omega}+\|\psi\|_{3-1 / q, q, \partial \Omega}\right)\right] .
\end{gathered}
$$

This proves Lemma 6.4 with $k=0$. Further, we proceed by induction to get

$$
\begin{gathered}
\lambda^{1 / 3}\left[\|\nabla u\|_{0,3 q /(3-q), \Omega}+\left\|\nabla^{2} u\right\|_{1, q, \Omega}+\|\nabla u\|_{k+1, p, \Omega}\right. \\
\left.+\|\nabla \Pi\|_{1, q, \Omega}+\|\Pi\|_{0,2 q /(2-q), \Omega}+\|\Pi\|_{k+1, p, \Omega}\right] \\
\leq c\left[\|\varpi\|_{1, q, \Omega}+\|\varpi\|_{k, p, \Omega}+\|h\|_{2, q, \Omega}+\|h\|_{k+1, p, \Omega}\right. \\
\left.+\lambda^{1 / 3}\left(\|\psi\|_{k+2-1 / p, p, \partial \Omega}+\|\psi\|_{3-1 / q, q, \partial \Omega}\right)\right] .
\end{gathered}
$$

Taking into account estimate (6.10), recalling that for $\lambda<\lambda_{0}$ (sufficiently small) and $1<q<6 / 5$,

$$
\lambda^{1 / 3}<|\ln \lambda|^{-1} \lambda^{2(1-1 / q)},
$$

we get estimate (6.12). Lemma 6.4 is thus proved. 


\section{References}

[1] S. Agmon, A. Douglis and L. Nirenberg, Estimates near the boundary of solutions of elliptic partial differential equations satisfying general boundary conditions II, Comm. Pure Appl. Math., 17 (1964), 35-92.

[2] R.A. Adams, Sobolev spaces, Academic Press (1976).

[3] Ch.J. Amick, On Leray's problem of steady Navier-Stokes flow past a body in the plane, Acta Math., 161 (1988), 71-130.

[4] Ch.J. Amick, On the asymptotic form of Navier-Stokes flow past a body in the plane, J. Diff. Eq., 91(1) (1991).

[5] K.I. Babenko, On stationary solutions of the problem of flow past a body of a viscous incompressible fluid, Mat. Sbornik, 91 (133) (1973) No. 1, 1-25.

[6] H. Beirão da Veiga, An $L^{p}$ - theory for the $n$-dimensional, stationary compressible Navier-Stokes equations and the incompressible limit for compressible fluids. The equilibrium solutions, Comm. Math. Phys., 109 (1987), 229-248.

[7] H. Beirão da Veiga, Boundary value problems for a class of first order partial differential equations in Sobolev spaces and applications to the Euler flow, Rend. Sem. Mat. Univ. Padova, 79 (1988), 247-273.

[8] W. Borchers and T. Miyakawa, Algebraic $L^{2}$-decay for the Navier-Stokes flows in exterior domains, Acta Math., 165 (1989), 189-227.

[9] W. Borchers and T. Miyakawa, On stability of exterior stationary Navier-Stokes flow, Acta Math., 174 (1995), 311-382.

[10] W. Borchers and H. Sohr, On the semigroup of the Stokes operator for exterior domains in $L^{q}$-spaces, Math. Z., 196 (1987), 415-425.

[11] L. Cattabriga, Su un problema al contorno relativo al systema di equazioni di Stokes, Rend. Sem. Mat. Padova, 31 (1961), 308-340.

[12] I.D. Chang and R. Finn, On the solutions of a class of equations occuring in continuum mechanics with applications to the Stokes paradox, Arch. Rat. Mech. Anal., 7 (1961), 388-441.

[13] D.C. Clark, The vorticity at infinity for solutions of the stationary Navier-Stokes equations in exterior domains, Indiana Univ. Math. J., 20 (1971), 633-654.

[14] R. Farwig, A variational approach in weighted Sobolev spaces to the operator $-\Delta+$ $\frac{\partial}{\partial x_{1}}$ in exterior domains of $\mathbb{R}^{3}$, Math. Z., 210 (1992), 449-464.

[15] R. Farwig, The stationary exterior 3-D problem of Oseen and Navier-Stokes equations in anisotropically weighted Sobolev spaces, Math. Z., 221 (1992), 406-447

[16] R. Farwig, Stationary solutions of the Navier-Stokes equations with slip boundary conditions, Comm. Part. Diff. Eq., 14 (1989), 1579-1606.

[17] R. Finn, On the exterior stationary problem for the Navier-Stokes equations and associated perturbation problems, Arch. Rat. Mech. Anal., 19 (1965), 363-406.

[18] R. Finn, On the steady state solutions of the Navier-Stokes equations, III, Acta Math., 105 (1961), 197-244.

[19] R. Finn, Mathematical questions relating to viscous fluid flow in an exterior domain, Rocky Mountain J. Math., 3(1) (1973), 107-140. 
[20] R. Finn and D.R. Smith, On the stationary solution of the Navier-Stokes equations in two dimensions, Arch. Rat. Mech. Anal., 25 (1967), 26-39.

[21] J. Fujita, On the existence and regularity of the steady-state solutions of the NavierStokes equations, J. Fac. Sci. Univ. Tokyo, Sect. IA, 9 (1961), 59-102.

[22] E. Gagliardo, Caratterizzazioni delle trace sulla frontiera relative ad alcune classi di funzioni in n variabili, Rend. Sem. Mat. Univ. Padova, 27 (1957), 284-305.

[23] G.P. Galdi, On the Oseen boundary value problem in exterior domains, Proc. of the Oberwolfach Meeting "The Navier-Stokes Equations: Theory and Numerical Methods", Heywood J.G., Masuda K., Rautman R., Solonnikov V.A. Eds., Lecture Notes in Mathematics, Springer (1991).

[24] G.P. Galdi, An introduction to the mathematical theory of the Navier Stokes equations - Vol. I, Vol. II, Springer Tracts in Natural Philosophy, Volumes 38 and 39 (1994).

[25] G.P. Galdi, On the asymptotic properties of Leray's solution to the exterior stationary three-dimensional Navier Stokes equations with zero velocity at infinity, I.M.A. Volumes in Mathematics and its Applications, Vol. 47, Springer-Verlag (1992), 95-104.

[26] G.P. Galdi, Existence and uniqueness at low Reynolds number of stationary plane flow of a viscous fluid in exterior domains, Pitman Research Notes in Mathematics Series, Vol. 291, (1993) 1-33.

[27] G.P. Galdi and Ch. Simader, Existence, Uniqueness and $L^{q}$-estimates for the Stokes problem in an exterior domain, Arch. Rat. Mech. Anal., 112 (1990), 231-318.

[28] D. Gilbarg and H.F. Weinberger, Asymptotic properties of Leray's solution of the stationary two-dimensional Navier-Stokes equations, Russian Math. Surveys, 29 (1974), 109-123.

[29] D. Gilbarg and H.F. Weinberger, Asymptotic properties of steady plane solutions of the Navier-Stokes equations with bounded Dirichlet integral, Ann. Scuola Norm. Sup. Pisa, 5(4) (1978), 381-404.

[30] J.G. Heywood, The Navier-Stokes equations: On the existence, regularity and decay of solutions, Indiana Univ. Math. J., 29(5) (1980), 639-681.

[31] H. Kozono and H. Sohr, New apriory estimates for the Stokes equation in exterior domains, Indiana Univ. Math. J., 40(1) (1991), 1-27.

[32] H. Kozono and H. Sohr, On a new class of generalized solutions for the Stokes equation in exterior domains, Ann. Sc. Norm. Sup. Pisa, 19 (1992), 155-181.

[33] H. Kozono and H. Sohr, On stationary Navier-Stokes equations in unbounded domains, Ricerche Mat., XLII(1) (1993), 69-86.

[34] J. Leray, Sur le mouvement d'une liquide visqueux emplissant l'espace, Acta Math., 63 (1934), 193-248.

[35] P. Maremonti and V.A. Solonnikov, Su una diseguaglianza per le soluzioni del problema di Stokes in domini esterni, preprint, Univ. Naples, (1983).

[36] A Matsumura, Fundamental solution of the linearized system for the exterior stationary problem of compressible viscous flow, in Pattern and Wawes, Qualitative Analysis of Nonlinear Differential Equations, (1986), 481-505.

[37] A. Matsumura and T. Nishida, Exterior stationary problems for the equations of motion of compressible viscous and heat conductive fluids, Proc. EQUADIFF 89, Ed. Dafermos, Ladas, Papanicolau, M. Dekker Inc. (1989), 473-479. 
[38] A. Matsumura and T. Nishida, Exterior stationary problems for the equations of motion of compressible viscous and heat conductive fluids, manuscript (in Japanese) (1989), 90p.

[39] A. Matsumura and T. Nishida, Initial boundary value problem for the equations of motion of compressible viscous and heat conductive fluids, Comm. Math. Phys., 89 (1983), 445-464.

[40] T. Miyakawa, On stationary solutions of the Navier-Stokes equations in an exterior domain, Hiroshima Math. J., 12 (1982),115-140.

[41] T. Miyakawa and H. Sohr, On energy inequality smoothness and large time behaviour of the Navier-Stokes equations in exterior domains, Math. Z., 193 (1988), 455-478.

[42] A. Novotný, About the steady transport equation $I-L^{p}$ - approach in domains with sufficiently smooth boundaries, Comm. Mat. Univ. Carolinae, 37(1) (1996), 43-89.

[43] A. Novotný, Steady flows of viscous compressible fluids $-L^{2}$-approach, Proc. of EQUAM 92, Salvi R., Straskraba I., Eds. SAACM, 3(3) (1993), 181-199.

[44] A. Novotný, Steady flows of viscous compressible fluids in exterior domains under small perturbations of great potential forces, Math. Meth. Model. Appl. Sci., 6(3) (1993), 725-757.

[45] A. Novotný and Padula M., $L^{p}$-approach to steady flows of viscous compressible fluids in exterior domains, Preprint Univ. Toulon, (1992), Arch. Rat. Mech. Anal., 126 (1994), 243-297.

[46] A. Novotný and M. Padula, Existence and uniqueness of stationary solutions for viscous compressible heat conductive fluid with large potential and small nonpotential external forces, Sib. Math. J., 34(5) (1993), 120-146.

[47] A. Novotny and M. Padula, Note on decay of solutions of steady Navier-Stokes equations in 3-D exterior domains, Diff. Int. Eq., 8(7) (1995), 1833-1842.

[48] A. Novotný and P. Penel, An $L^{p}$-approach for steady flows of viscous compressible heat conductive gas I, II, Math. Meth. Model. Appl. Sci., (in press).

[49] M. Padula, On the exterior steady problem for the equations of a viscous isothermal gas, Com. Mat. Univ. Carolinae, 34(2) (1993), 275-293.

[50] M. Padula, A representation formula for steady solutions of a compressible fluid moving at low speed, Transp. Th. Stat. Phys., 21 (1993), 593-613.

[51] M. Padula, Existence and uniqueness for viscous steady compressible motions, Arch. Rat. Mech. Anal., 77(2) (1987), 89-102.

[52] Ch. Simader, The weak Dirichlet and Neumann problem for the Laplacian in $L^{q}$ for bounded and exterior domains. Applications in Nonlinear analysis, function spaces and applications, editors Krbec, Kufner, Opic, Rakosnik, Vol. 4, Leipzig, Teubner, (1990), 180-223.

[53] Ch. Simader and H. Sohr, The weak Dirichlet problem for $\Delta$ in $L^{q}$ in bounded and exterior domains, Pitman Research Notes in Mathematics, (in press).

[54] Ch. Simader and H. Sohr, A new approach to the Helmholtz decomposition and the Neumann problem in $L^{q}$-spaces for bounded and exterior domains, in Mathematical problems relating to Navier-Stokes equations, Series of Advances in Mathematics for Applied Sciences, Vol. 11, Editor G.P. Galdi, World Scientific (1992).

[55] D.R. Smith, Estimates at infinity for stationary solutions of the Navier-Stokes equations in two dimensions, Arch. Rat. Mech. Anal., 20 (1965), 341-372. 
[56] H. Sohr and W. von Wahl, On the singular set and the uniqueness of the NavierStokes equations, Manuscripta Mat., 49 (1984), 27-59.

[57] A. Valli, On the existence of stationary solutions to compressible Navier-Stokes equations, Ann. Inst. H. Poincaré, 4(1) (1987), 99-113.

[58] E. Zeidler, Nonlinear Functional Analysis and Applications I, Springer (1986).

Received January 6, 1995.

ISTITUTE OF ENGINEERING

UNIVERSITY OF FERRARA

ITALY

AND

UNIVERSITY OF TOULON AND OF VAR

La Garde, France

AND

UNIVERSITY OF FERRARA

ITALY 